\title{
From molecular dynamics and particle simulations towards constitutive relations for continuum theory
}

Stefan Luding

\begin{abstract}
One challenge of todays research is the realistic simulation of disordered atomistic systems or particulate and granular materials like sand, powders, ceramics or composites, which consist of many millions of atoms/particles. The inhomogeneous fine-structure of such materials makes it very difficult to treat them with continuum methods, which typically assume homogeneity and scale separation. As an alternative, particle based methods can be straightforwardly applied, since they intrinsically take the fine-structure into account. The ultimate challenge is to find constitutive relations for continuum theory from these particle-based simulations. In this chapter, a particle simulation approach, the so-called discrete element method (DEM), as related to molecular dynamics (MD) methods, is introduced and applied to the simulation of many-particle systems. The examples (clustering in granular gases, and bi-axial as well as cylindrical shearing of dense packings) illustrate the micro-macro transition towards continuum theory.

There exist two basically different approaches, the so-called soft particle molecular dynamics and the hard sphere, event-driven method. The former is straightforward, easy to generalize, and has numberless applications, while the latter is optimized for rigid interactions and is mainly used for collisional, dissipative granular gases. The connection between the two methods will be elaborated on. Models for the forces between the atoms/particles are the basis of both MD and DEM. A set of the most basic contact force models for particles is presented involving elastoplasticity, adhesion, viscosity, static and dynamic friction as well as rolling- and torsion-resistance. Besides some words about van-der Waals forces, we will not detail on electro-magnetic interactions, dipole moments, H-bonding, and other effects which become important when the objects become smaller and smaller.
\end{abstract}

Key words: molecular dynamics (MD), discrete element methods (DEM), event driven $\mathrm{MD}$, equation of state, clustering, shear band formation, micro-macro

Multi Scale Mechanics, TS, CTW, UTwente, P.O.Box 217, 7500 AE Enschede, Netherlands e-mail: s.luding@utwente.nl -- www2.msm.ctw.utwente.nl/sluding 


\section{Introduction}

Materials with inhomogeneous fine-structures are the subject of this chapter. As example, we mostly discuss particulate, granular systems where the fine-structures are spherical, polydisperse, plastic, adhesive, and frictional objects.

One approach towards the microscopic understanding of such macroscopic particulate material behavior $[19,25,20]$ is the modeling of particles using so-called discrete element methods (DEM). Even though millions of particles can be simulated, the possible length of such a particle system is in general too small in order to regard it as macroscopic. Therefore, methods and tools to perform a so-called micro-macro transition $[68,58,24]$ are discussed, starting from the DEM simulations. These "microscopic" simulations of a small sample (representative volume element) can be used to derive macroscopic constitutive relations needed to describe the material within the framework of a macroscopic continuum theory.

For granular materials, as an example, the particle properties and interaction laws are inserted into DEM, which is also often referred to as molecular dynamics (MD), and lead to the collective behavior of the dissipative many-particle system. From a particle simulation, one can extract, e.g., the pressure of the system as a function of density. This equation of state allows a macroscopic description of the material, which can be viewed as a compressible, non-Newtonian complex fluid [48], including a fluid-solid phase transition.

In the following, two versions of the molecular dynamics simulation method are introduced. The first is the so-called soft sphere molecular dynamics (MD=DEM), as described in section 2. It is a straightforward implementation to solve the equations of motion for a system of many interacting particles [5, 59]. For DEM, both normal and tangential interactions, like friction, are discussed for spherical particles. The second method is the so-called event-driven (ED) simulation, as discussed in section 3, which is conceptually different from DEM, since collisions are dealt with via a collision matrix that determines the momentum change on physical grounds. For the sake of brevity, the ED method is only discussed for smooth spherical particles. A comparison and a way to relate the soft and hard particle methods is provided in section 4.

As one ingredient of a micro-macro transition, the stress is defined for a dynamic system of hard spheres, in section 5, by means of kinetic-theory arguments [58], and for a quasi-static system by means of volume averages [26]. Examples are presented in the following sections 6 and 7, where the above-described methods are applied.

\section{The Soft Particle Molecular Dynamics Method}

One possibility to obtain information about the behavior of granular media is to perform experiments. An alternative are simulations with the molecular dynamics (MD) or discrete element model (DEM) [68, 9, 8, 6, 19, 63, 64, 65, 27]. Note that 
both methods are identical in spirit, however, different groups of researchers use these (and also other) names.

Conceptually, the DEM method has to be separated from the hard sphere eventdriven (ED) molecular dynamics, see section 3, and also from the so-called Contact Dynamics (CD). Like alternative (stochastic) methods, as there are cell- or latticegas-methods these are just named as keywords - not discussed here further.

\subsection{Discrete Particle Model}

The elementary units of granular materials are mesoscopic grains which deform under stress. Since the realistic modeling of the deformations of the particles is much too complicated, we relate the interaction force to the overlap $\delta$ of two particles, see Fig. 1. Note that the evaluation of the inter-particle forces based on the overlap may not be sufficient to account for the inhomogeneous stress distribution inside the particles. Consequently, our results presented below are of the same quality as the simple assumptions about the force-overlap relation, see Fig. 1.
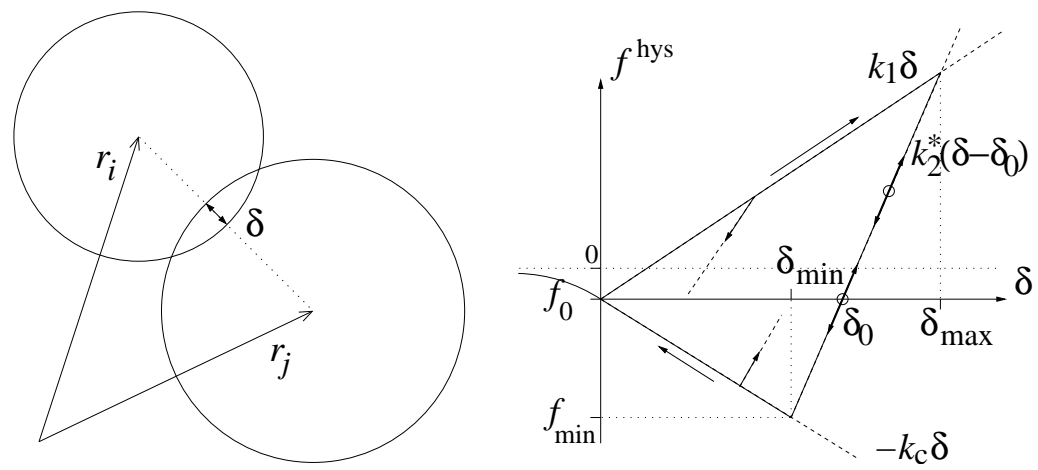

Fig. 1 (Left) Two particle contact with overlap $\delta$. (Right) Schematic graph of the piecewise linear, hysteretic, adhesive force-displacement model used below.

\subsection{Equations of Motion}

If all forces $f_{i}$ acting on the particle $i$, either from other particles, from boundaries or from external forces, are known, the problem is reduced to the integration of Newton's equations of motion for the translational and rotational degrees of freedom: 


$$
m_{i} \frac{\mathrm{d}^{2}}{\mathrm{~d} t^{2}} r_{i}=f_{i}+m_{i} g, \text { and } I_{i} \frac{\mathrm{d}^{2}}{\mathrm{~d} t^{2}} \varphi_{i}=t_{i}
$$

with the mass $m_{i}$ of particle $i$, its position $r_{i}$ the total force $f_{i}=\sum_{c} f_{i}^{c}$ acting on it due to contacts with other particles or with the walls, the acceleration due to volume forces like gravity $g$, the spherical particles moment of inertia $I_{i}$, its angular velocity $\omega_{i}=\mathrm{d} \varphi_{i} / \mathrm{d} t$ and the total torque $t_{i}=\sum_{c}\left(l_{i}^{c} \times f_{i}^{c}+q_{i}^{c}\right)$, where $q_{i}^{c}$ are torques/couples at contacts other than due to a tangential force, e.g., due to rolling and torsion.

The equations of motion are thus a system of $\mathscr{D}+\mathscr{D}(\mathscr{D}-1) / 2$ coupled ordinary differential equations to be solved in $\mathscr{D}$ dimensions. With tools from numerical integration, as nicely described in textbooks as [5, 59], this is straightforward. The typically short-ranged interactions in granular media, allow for a further optimization by using linked-cell or alternative methods $[5,59]$ in order to make the neighborhood search more efficient. In the case of long-range interactions, (e.g. charged particles with Coulomb interaction, or objects in space with self-gravity) this is not possible anymore, so that more advanced methods for optimization have to be applied - for the sake of brevity, we restrict ourselves to short range interactions here.

\subsection{Normal Contact Force Laws}

\subsubsection{Linear Normal Contact Model}

Two spherical particles $i$ and $j$, with radii $a_{i}$ and $a_{j}$, respectively, interact only if they are in contact so that their overlap

$$
\delta=\left(a_{i}+a_{j}\right)-\left(r_{i}-r_{j}\right) \cdot n
$$

is positive, $\delta>0$, with the unit vector $n=n_{i j}=\left(r_{i}-r_{j}\right) /\left|r_{i}-r_{j}\right|$ pointing from $j$ to $i$. The force on particle $i$, from particle $j$, at contact $c$, can be decomposed into a normal and a tangential part as $f^{c}:=f_{i}^{c}=f^{n} n+f^{t} t$, where $f^{n}$ is discussed first.

The simplest normal contact force model, which takes into account excluded volume and dissipation, involves a linear repulsive and a linear dissipative force

$$
f^{n}=k \delta+\gamma_{0} v_{n}
$$

with a spring stiffness $k$, a viscous damping $\gamma_{0}$, and the relative velocity in normal direction $v_{n}=-v_{i j} \cdot n=-\left(v_{i}-v_{j}\right) \cdot n=\dot{\delta}$.

This so-called linear spring dashpot model allows to view the particle contact as a damped harmonic oscillator, for which the half-period of a vibration around an equilibrium position, see Fig. 1, can be computed, and one obtains a typical response time on the contact level,

$$
t_{c}=\frac{\pi}{\omega}, \text { with } \omega=\sqrt{\left(k / m_{12}\right)-\eta_{0}^{2}},
$$


with the eigenfrequency of the contact $\omega$, the rescaled damping coefficient $\eta_{0}=$ $\gamma_{0} /\left(2 m_{i j}\right)$, and the reduced mass $m_{i j}=m_{i} m_{j} /\left(m_{i}+m_{j}\right)$. From the solution of the equation of a half period of the oscillation, one also obtains the coefficient of restitution

$$
r=v_{n}^{\prime} / v_{n}=\exp \left(-\pi \eta_{0} / \omega\right)=\exp \left(-\eta_{0} t_{c}\right),
$$

which quantifies the ratio of relative velocities after (primed) and before (unprimed) the collision.

The contact duration in Eq. (4) is also of practical technical importance, since the integration of the equations of motion is stable only if the integration timestep $\Delta t_{\mathrm{DEM}}$ is much smaller than $t_{c}$. Furthermore, it depends on the magnitude of dissipation. In the extreme case of an overdamped spring, $t_{c}$ can become very large. Therefore, the use of neither too weak nor too strong dissipation is recommended.

\subsubsection{Adhesive, Elasto-Plastic Normal Contact Model}

Here we apply a variant of the linear hysteretic spring model [69, 31, 67, 39], as an alternative to the frequently applied spring-dashpot models. This model is the simplest version of some more complicated nonlinear-hysteretic force laws $[69,70$, 60], which reflect the fact that at the contact point, plastic deformations may take place. The repulsive (hysteretic) force can be written as

$$
f^{\text {hys }}=\left\{\begin{array}{lll}
k_{1} \delta & \text { for loading, } & \text { if } k_{2}^{*}\left(\delta-\delta_{0}\right) \geq k_{1} \delta \\
k_{2}^{*}\left(\delta-\delta_{0}\right) & \text { for un/reloading, } & \text { if } k_{1} \delta>k_{2}^{*}\left(\delta-\delta_{0}\right)>-k_{c} \delta \\
-k_{c} \delta & \text { for unloading, } & \text { if }-k_{c} \delta \geq k_{2}^{*}\left(\delta-\delta_{0}\right)
\end{array}\right.
$$

with $k_{1} \leq k_{2}^{*}$, see Fig. 1, and Eq. (7) below for the definition of the (variable) $k_{2}^{*}$ as function of the constant model parameter $k_{2}$.

During the initial loading the force increases linearly with the overlap $\delta$, until the maximum overlap $\delta_{\max }$ is reached (which has to be kept in memory as a history parameter). The line with slope $k_{1}$ thus defines the maximum force possible for a given $\delta$. During unloading the force drops from its value at $\delta_{\max }$ down to zero at overlap $\delta_{0}=\left(1-k_{1} / k_{2}^{*}\right) \delta_{\max }$, on the line with slope $k_{2}^{*}$. Reloading at any instant leads to an increase of the force along this line, until the maximum force is reached; for still increasing $\delta$, the force follows again the line with slope $k_{1}$ and $\delta_{\max }$ has to be adjusted accordingly.

Unloading below $\delta_{0}$ leads to negative, attractive forces until the minimum force $-k_{c} \delta_{\min }$ is reached at the overlap $\delta_{\min }=\left(k_{2}^{*}-k_{1}\right) \delta_{\max } /\left(k_{2}^{*}+k_{c}\right)$. This minimum force, i.e. the maximum attractive force, is obtained as a function of the model parameters $k_{1}, k_{2}, k_{c}$, and the history parameter $\delta_{\text {max }}$. Further unloading leads to attractive forces $f^{\text {hys }}=-k_{c} \delta$ on the adhesive branch with slope $-k_{c}$. The highest possible attractive force, for given $k_{1}$ and $k_{2}$, is reached for $k_{c} \rightarrow \infty$, so that $f_{\max }^{\text {hys }}=-\left(k_{2}-k_{1}\right) \delta_{\max }$. Since this would lead to a discontinuity at $\delta=0$, it is avoided by using finite $k_{c}$. 
The lines with slope $k_{1}$ and $-k_{c}$ define the range of possible force values and departure from these lines takes place in the case of unloading and reloading, respectively. Between these two extremes, unloading and reloading follow the same line with slope $k_{2}$. Possible equilibrium states are indicated as circles in Fig. 1, where the upper and lower circle correspond to a pre-stressed and stress-free state, respectively. Small perturbations lead, in general, to small deviations along the line with slope $k_{2}$ as indicated by the arrows.

A non-linear un/reloading behavior would be more realistic, however, due to a lack of detailed experimental informations, we use the piece-wise linear model as a compromise. One refinement is a $k_{2}^{*}$ value dependent on the maximum overlap that implies small and large plastic deformations for weak and strong contact forces, respectively. One model, as implemented recently [50, 39], requires an additional model parameter, $\delta_{\max }^{*}$, so that $k_{2}^{*}\left(\delta_{\max }\right)$ is increasing from $k_{1}$ to $k_{2}$ (linear interpolation is used below, however, this is another choice to be made and will depend on the material under consideration) with the maximum overlap, until $\delta_{\max }^{*}$ is reached 1 :

$$
k_{2}^{*}\left(\delta_{\max }\right)=\left\{\begin{array}{ll}
k_{2} & \text { if } \delta_{\max } \geq \delta_{\max }^{*} \\
k_{1}+\left(k_{2}-k_{1}\right) \delta_{\max } / \delta_{\max }^{*} & \text { if } \delta_{\max }<\delta_{\max }^{*}
\end{array} .\right.
$$

While in the case of collisions of particles with large deformations, dissipation takes place due to the hysteretic nature of the force-law, stronger dissipation of small amplitude deformations is achieved by adding the viscous, velocity dependent dissipative force from Eq. (3) to the hysteretic force, such that $f^{n}=f^{\text {hys }}+\gamma_{0} v_{n}$. The hysteretic model contains the linear contact model as special case $k_{1}=k_{2}=k$.

\subsubsection{Long Range Normal Forces}

Medium range van der Waals forces can be taken into account in addition to the hysteretic force such that $f^{n}=f_{i}^{\text {hys }}+f_{i}^{\mathrm{vdW}}$ with, for example, the attractive part of a Lennard-Jones Potential

$$
f^{\mathrm{vdW}}=-6\left(\varepsilon / r_{0}\right)\left[\left(r_{0} / r_{i j}\right)^{7}-\left(r_{0} / r_{c}\right)^{7}\right] \text { for } r_{i j} \leq r_{c} .
$$

The new parameters necessary for this force are an energy scale $\varepsilon$, a typical length scale $r_{0}$ and a cut-off length $r_{c}$. As long as $r_{c}$ is not much larger than the particle diameter, the methods for short range interactions still can be applied to such a medium range interaction model - only the linked cells have to be larger than twice the cut-off radius, and no force is active for $r>r_{c}$.

\footnotetext{
${ }^{1}$ A limit to the slope $k_{2}$ is needed for practical reasons. If $k_{2}$ would not be limited, the contact duration could become very small so that the time step would have to be reduced below reasonable values.
} 


\subsection{Tangential Forces and Torques in General}

For the tangential degrees of freedom, there are three different force- and torquelaws to be implemented: (i) friction, (ii) rolling resistance, and (iii) torsion resistance.

\subsubsection{Sliding}

For dynamic (sliding) and static friction, the relative tangential velocity of the contact points,

$$
v_{t}=v_{i j}-n\left(n \cdot v_{i j}\right),
$$

is to be considered for the force and torque computations in subsection 2.5, with the total relative velocity of the particle surfaces at the contact

$$
v_{i j}=v_{i}-v_{j}+a_{i}^{\prime} n \times \omega_{i}+a_{j}^{\prime} n \times \omega_{j},
$$

with the corrected radius relative to the contact point $a_{\alpha}^{\prime}=a_{\alpha}-\delta / 2$, for $\alpha=i, j$. Tangential forces acting on the contacting particles are computed from the accumulated sliding of the contact points along each other, as described in detail in subsection 2.5.1.

\subsubsection{Objectivity}

In general, two particles can rotate together, due to both a rotation of the reference frame or a non-central "collision". The angular velocity $\omega_{0}=\omega_{0}^{n}+\omega_{0}^{t}$, of the rotating reference has the tangential-plane component

$$
\omega_{0}^{t}=\frac{n \times\left(v_{i}-v_{j}\right)}{a_{i}^{\prime}+a_{j}^{\prime}},
$$

which is related to the relative velocity, while the normal component, $\omega_{0}^{n}$, is not. Inserting $\omega_{i}=\omega_{j}=\omega_{0}^{t}$, from Eq. (11), into Eq. (10) leads to zero sliding velocity, proving that the above relations are objective. Tangential forces and torques due to sliding can become active only when the particles are rotating with respect to the common rotating reference frame. ${ }^{2}$

Since action should be equal to reaction, the tangential forces are equally strong, but opposite, i.e., $f_{j}^{t}=-f_{i}^{t}$, while the corresponding torques are parallel but not necessarily equal in magnitude: $q_{i}^{\text {friction }}=-a_{i}^{\prime} n \times f_{i}$, and $q_{j}^{\text {friction }}=\left(a_{j}^{\prime} / a_{i}^{\prime}\right) q_{i}^{\text {friction }}$. Note that tangential forces and torques together conserve the total angular momen-

\footnotetext{
${ }^{2}$ For rolling and torsion, there is no similar relation between rotational and tangential degrees of freedom: for any rotating reference frame, torques due to rolling and torsion can become active only due to rotation relative to the common reference frame, see below.
} 
tum about the pair center of mass

$$
L_{i j}=L_{i}+L_{j}+m_{i} r_{i \mathrm{~cm}}^{2} \omega_{0}^{t}+m_{j} r_{j \mathrm{~cm}}^{2} \omega_{0}^{t},
$$

with the rotational contributions $L_{\alpha}=I_{\alpha} \omega_{\alpha}$, for $\alpha=i, j$, and the distances $r_{\alpha \mathrm{cm}}=$ $\left|r_{\alpha}-r_{\mathrm{cm}}\right|$ from the particle centers to the center of mass $r_{\mathrm{cm}}=\left(m_{i} r_{i}+m_{j} r_{j}\right) /\left(m_{i}+\right.$ $\left.m_{j}\right)$, see Ref. [31]. The change of angular momentum consists of the change of particle spins (first term) and of the change of the angular momentum of the two masses rotating about their common center of mass (second term):

$$
\frac{d L_{i j}}{d t}=q_{i}^{\text {friction }}\left(1+\frac{a_{j}^{\prime}}{a_{i}^{\prime}}\right)+\left(m_{i} r_{i \mathrm{~cm}}^{2}+m_{j} r_{j \mathrm{~cm}}^{2}\right) \frac{d \omega_{0}^{t}}{d t},
$$

which both contribute, but exactly cancel each other, since

$$
\begin{aligned}
q_{i}^{\text {friction }}\left(1+\frac{a_{j}^{\prime}}{a_{i}^{\prime}}\right) & =-\left(a_{i}^{\prime}+a_{j}^{\prime}\right) n \times f_{i} \\
& =-\left(m_{i} r_{i \mathrm{~cm}}^{2}+m_{j} r_{j \mathrm{~cm}}^{2}\right) \frac{d \omega_{0}^{t}}{d t}
\end{aligned}
$$

see [37] for more details.

\subsubsection{Rolling}

A rolling velocity $v_{r}^{0}=-a_{i}^{\prime} n \times \omega_{i}+a_{j}^{\prime} n \times \omega_{j}$, defined in analogy to the sliding velocity, is not objective in general $[14,37]$ - only in the special cases of (i) equalsized particles or (ii) for a particle rolling on a fixed flat surface.

The rolling velocity should quantify the distance the two surfaces roll over each other (without sliding). Therefore, it is equal for both particles by definition. An objective rolling velocity is obtained by using the reduced radius, $a_{i j}^{\prime}=a_{i}^{\prime} a_{j}^{\prime} /\left(a_{i}^{\prime}+\right.$ $\left.a_{j}^{\prime}\right)$, so that

$$
v_{r}=-a_{i j}^{\prime}\left(n \times \omega_{i}-n \times \omega_{j}\right) .
$$

This definition is objective since any common rotation of the two particles vanishes by construction. A more detailed discussion of this issue is beyond the scope of this paper, rather see $[14,37]$ and the references therein.

A rolling velocity will activate torques, acting against the rolling motion, e.g., when two particles are rotating anti-parallel with spins in the tangential plane. These torques are then equal in magnitude and opposite in direction, i.e., $q_{i}^{\text {rolling }}=$ $-q_{j}^{\text {rolling }}=a_{i j} n \times f_{r}$, with the quasi-force $f_{r}$, computed in analogy to the friction force, as function of the rolling velocity $v_{r}$ in subsection 2.5.2; the quasi-forces for both particles are equal and do not act on the centers of mass. Therefore, the total momenta (translational and angular) are conserved. 


\subsubsection{Torsion}

For torsion resistance, the relative spin along the normal direction

$$
v_{o}=a_{i j}\left(n \cdot \omega_{i}-n \cdot \omega_{j}\right) n,
$$

is to be considered, which activates torques when two particles are rotating antiparallel with spins parallel to the normal direction. Torsion is not activated by a common rotation of the particles around the normal direction $n \cdot \omega_{0}=n \cdot\left(\omega_{i}+\omega_{j}\right) / 2$, which makes the torsion resistance objective.

The torsion torques are equal in magnitude and directed in opposite directions, i.e., $q_{i}^{\text {torsion }}=-q_{j}^{\text {torsion }}=a_{i j} f_{o}$, with the quasi-force $f_{o}$, computed from the torsion velocity in subsection 2.5 .3 , and also not changing the translational momentum. Like for rolling, the torsion torques conserve the total angular momentum.

\subsubsection{Summary}

The implementation of the tangential force computations for $f_{t}, f_{r}$, and $f_{o}$ as based on $v_{t}, v_{r}$, and $v_{o}$, respectively, is assumed to be identical, i.e., even the same subroutine is used, but with different parameters as specified below. The difference is that friction leads to a force in the tangential plane (changing both translational and angular momentum), while rolling- and torsion-resistance lead to quasi-forces in the tangential plane and the normal direction, respectively, changing the particles' angular momentum only. For more details on tangential contact models, friction, rolling and torsion, see Refs. [7, 13, 38, 37, 14].

\subsection{The tangential force- and torque-models}

The tangential contact model presented now is a single procedure (subroutine) that can be used to compute either sliding, rolling, or torsion resistance. The subroutine needs a relative velocity as input and returns the respective force or quasi-force as function of the accumulated deformation. The sliding/sticking friction model will be introduced in detail, while rolling and torsion resistance are discussed where different.

\subsubsection{Sliding/Sticking Friction Model}

The tangential force is coupled to the normal force via Coulomb's law, $f^{t} \leq f_{C}^{s}:=$ $\mu^{s} f^{n}$, where for the sliding case one has dynamic friction with $f^{t}=f_{C}^{t}:=\mu^{d} f^{n}$. The dynamic and the static friction coefficients follow, in general, the relation $\mu^{d} \leq \mu^{s}$. The static situation requires an elastic spring in order to allow for a restoring force, 
i.e., a non-zero remaining tangential force in static equilibrium due to activated Coulomb friction.

If a purely repulsive contact is established, $f^{n}>0$, and the tangential force is active. For an adhesive contact, Coulombs law has to be modified in so far that $f^{n}$ is replaced by $f^{n}+k_{c} \delta$. In this model, the reference for a contact is no longer the zero force level, but it is the adhesive, attractive force level along $-k_{c} \delta$.

If a contact is active, one has to project (or better rotate) the tangential spring into the actual tangential plane, since the frame of reference of the contact may have rotated since the last time-step. The tangential spring

$$
\xi=\xi^{\prime}-n\left(n \cdot \xi^{\prime}\right)
$$

is used for the actual computation, where $\xi^{\prime}$ is the old spring from the last iteration, with $|\xi|=\left|\xi^{\prime}\right|$ enforced by appropriate scaling/rotation. If the spring is new, the tangential spring-length is zero, but its change is well defined after the first, initiation step. In order to compute the changes of the tangential spring, a tangential test-force is first computed as the sum of the tangential spring force and a tangential viscous force (in analogy to the normal viscous force)

$$
f_{0}^{t}=-k_{t} \xi-\gamma_{t} v_{t}
$$

with the tangential spring stiffness $k_{t}$, the tangential dissipation parameter $\gamma_{t}$, and $v_{t}$ from Eq. (9). As long as $\left|f_{0}^{t}\right| \leq f_{C}^{s}$, with $f_{C}^{s}=\mu^{s}\left(f^{n}+k_{c} \delta\right)$, one has static friction and, on the other hand, for $\left|f_{0}^{t}\right|>f_{C}^{s}$, sliding friction becomes active. As soon as $\left|f_{0}^{t}\right|$ gets smaller than $f_{C}^{d}$, static friction becomes active again.

In the static friction case, below the Coulomb limit, the tangential spring is incremented

$$
\xi^{\prime}=\xi+v_{t} \Delta t_{\mathrm{MD}},
$$

to be used in the next iteration in Eq. (17), and the tangential force $f^{t}=f_{0}^{t}$ from Eq. (18) is used. In the sliding friction case, the tangential spring is adjusted to a length consistent with Coulombs condition, so that

$$
\xi^{\prime}=-\frac{1}{k_{t}}\left(f_{C}^{d} t+\gamma_{t} v_{t}\right)
$$

with the tangential unit vector, $t=f_{0}^{t} /\left|f_{0}^{t}\right|$, defined by Eq. (18), and thus the magnitude of the Coulomb force is used. Inserting $\xi^{\prime}$ from Eq. (20) into Eq. (18) during the next iteration will lead to $f_{0}^{t} \approx f_{C}^{d} t$. Note that $f_{0}^{t}$ and $v_{t}$ are not necessarily parallel in three dimensions. However, the mapping in Eq. (20) works always, rotating the new spring such that the direction of the frictional force is unchanged and, at the same time, limiting the spring in length according to Coulombs law. In short notation the tangential contact law reads

$$
f^{t}=f^{t} t=+\min \left(f_{C},\left|f_{0}^{t}\right|\right) t,
$$


where $f_{C}$ follows the static/dynamic selection rules described above. The torque on a particle due to frictional forces at this contact is $q^{\text {friction }}=l_{i}^{c} \times f_{i}^{c}$, where $l_{i}^{c}$ is the branch vector, connecting the center of the particle with the contact point. Note that the torque on the contact partner is generally different in magnitude, since $l_{i}^{c}$ can be different, but points in the same direction; see subsection 2.4.2 for details on this.

The four parameters for the friction law are $k_{t}, \mu_{s}, \phi_{d}=\mu_{d} / \mu_{s}$, and $\gamma_{t}$, accounting for tangential stiffness, the static friction coefficient, the dynamic friction ratio, and the tangential viscosity, respectively. Note that the tangential force described above is identical to the classical Cundall-Strack spring only in the limits $\mu=\mu^{s}=\mu^{d}$, i.e., $\phi_{d}=1$, and $\gamma_{t}=0$. The sequence of computations and the definitions and mappings into the tangential direction can be used in $3 \mathrm{D}$ as well as in $2 \mathrm{D}$.

\subsubsection{Rolling Resistance Model}

The three new parameters for rolling resistance are $k_{r}, \mu_{r}$, and $\gamma_{r}$, while $\phi_{r}=\phi_{d}$ is used from the friction law. The new parameters account for rolling stiffness, a static rolling "friction" coefficient, and rolling viscosity, respectively. In the subroutine called, the rolling velocity $v_{r}$ is used instead of $v_{t}$ and the computed quasi-force $f_{r}$ is used to compute the torques, $q^{\text {rolling }}$, on the particles.

\subsubsection{Torsion Resistance Model}

The three new parameters for rolling resistance are $k_{o}, \mu_{o}$, and $\gamma_{o}$, while $\phi_{o}=\phi_{d}$ is used from the friction law. The new parameters account for torsion stiffness, a static torsion "friction" coefficient, and torsion viscosity, respectively. In the subroutine, the torsion velocity $v_{o}$ is used instead of $v_{t}$ and the projection is a projection along the normal unit-vector, not into the tangential plane as for the other two models. The computed quasi-force $f_{o}$ is then used to compute the torques, $q^{\text {torsion }}$, on the particles.

\subsection{Background Friction}

Note that the viscous dissipation takes place in a two-particle contact. In the bulk material, where many particles are in contact with each other, this dissipation mode is very inefficient for long-wavelength cooperative modes of motion [42, 41]. Therefore, an additional damping with the background can be introduced, so that the total force on particle $i$ is

$$
f_{i}=\sum_{j}\left(f^{n} n+f^{t} t\right)-\gamma_{b} v_{i}
$$

and the total torque 


$$
q_{i}=\sum_{j}\left(q^{\text {friction }}+q^{\text {rolling }}+q^{\text {torsion }}\right)-\gamma_{b r} a_{i}^{2} \omega_{i}
$$

with the damping artificially enhanced in the spirit of a rapid relaxation and equilibration. The sum in Eqs. (22) and (23) takes into account all contact partners $j$ of particle $i$, but the background dissipation can be attributed to the medium between the particles. Note that the effect of $\gamma_{b}$ and $\gamma_{b r}$ should be checked for each set of parameters: it should be small in order to exclude artificial over-damping. The set of parameters is summarized in table 1 . Note that only a few parameters are specified with dimensions, while the other paramters are expressed as ratios.

\begin{tabular}{|l|l|}
\hline Property & Symbol \\
\hline \hline Time unit & $t_{u}$ \\
Length unit & $x_{u}$ \\
Mass unit & $m_{u}$ \\
\hline Particle radius & $a_{0}$ \\
Material density & $\rho$ \\
\hline Elastic stiffness (variable) & $k_{2}$ \\
Maximal elastic stiffness & $k=k_{2}$ \\
Plastic stiffness & $k_{1} / k$ \\
Adhesion "stiffness" & $k_{c} / k$ \\
Friction stiffness & $k_{t} / k$ \\
Rolling stiffness & $k_{r} / k$ \\
Torsion stiffness & $k_{o} / k$ \\
\hline Plasticity depth & $\phi_{f}$ \\
\hline Coulomb friction coefficient & $\mu=\mu_{d}=\mu_{s}$ \\
Dynamic to static Friction ratio & $\phi_{d}=\mu_{d} / \mu_{s}$ \\
Rolling "friction" coefficient & $\mu_{r}$ \\
Torsion "friction" coefficient & $\mu_{o}$ \\
\hline Normal viscosity & $\gamma=\gamma_{n}$ \\
Friction viscosity & $\gamma_{t} / \gamma$ \\
Rolling viscosity & $\gamma_{r} / \gamma$ \\
Torsion viscosity & $\gamma_{o} / \gamma$ \\
Background viscosity & $\gamma_{b} / \gamma$ \\
Background viscous torque & $\gamma_{b r} / \gamma$ \\
\hline
\end{tabular}

Table 1 Summary of the microscopic contact model parameters. The longer ranged forces and their parameters, $\varepsilon, r_{0}$, and $r_{c}$ are not included here.

\subsection{Example: Tension Test Simulation Results}

In order to illustrate the power of the contact model (especially the adhesive normal model), in this section, uni-axial tension and compression tests are presented. Note that the contact model parameters are chosen once and then one can simulate loose particles, pressure-sintering, and agglomerates with one set of paramters. With slight 
extensions, the same model was already applied to temperature-sintering [50] or self-healing $[53,52]$.

The tests consists of three stages: (i) pressure sintering, (ii) stress-relaxation, and (iii) the compression- or tension-test itself. The contact parameters, as introduced in the previous section, are summarized in table 1 and typical values are given in table 2. These parameters are used for particle-particle contacts, the same for all tests, unless explicitly specified.

First, for pressure sintering, a very loose assembly of particles is compressed with isotropic stress $p_{s} 2 a / k_{2} \approx 0.02$ in a cuboidal volume so that the adhesive contact forces are activated this way. The stress- and strain-controlled wall motion modes aredescribed below in subsection 6.2.2.

Two of the six walls are adhesive, with $k_{c}^{\text {wall }} / k_{2}=20$, so that the sample sticks to them later, while all other walls are adhesionless, so that they can be easily removed in the next step. Note that during compression and sintering, the walls could all be without adhesion, since the high pressure used keeps the sample together anyway - only later for relaxation, adhesion must switched on. If not the sample does not remain a solid, and it also could lose contact with the walls, which are later used to apply the tensile strain.

All walls should be frictionless during sintering, while the particles can be slightly adhesive and frictional. If the walls would be frictional, the pressure from a certain wall would not be transferred completely to the respective opposite wall, since frictional forces carry part of the load - an effect that is known since the early work of Janssen [21, 62, 66].

Pressure-sintering is stopped when the kinetic energy of the sample is many orders of magnitude smaller than the potential energy - typically 10 orders of magnitude.

During stress-relaxation all wall stresses are slowly released to $p_{r} / p_{s} \ll 1$ and the sample is relaxed again until the kinetic energy is much smaller than the potential energy. After this, the sample is ready for the tension or compression tests. The nonadhesive side walls still feel a very small external stress that is not big enough to affect the dynamics of the tension test, it is just convenient to keep the walls close to the sample. (This is a numerical and not a physical requirement, since our code uses linked-cells and those are connected to the system size. If the walls would move too far away, either the linked cells would grow, or their number would increase. Both cases are numerically inefficient.)

For the tension test wall friction is typically active, but some variation does not show a big effect. One of the sticky walls is slowly and smoothly moved outwards like described and applied in earlier studies [46, 35, 38, 53, 39, 52], following a prescribed cosine-function with time.

\subsubsection{Model Parameters for tension}

The system presented in this subsection contains $N=1728$ particles with radii $a_{i}$ drawn from a Gaussian distribution around $a=0.005 \mathrm{~mm}[11,10]$. The contact model parameters are summarized in tables 1 and 2 . The volume fraction, $v=$ 
$\sum_{i} V\left(a_{i}\right) / V$, with the particle volume $V\left(a_{i}\right)=(4 / 3) \pi a_{i}^{3}$, reached during pressure sintering with $2 a p_{s} / k_{2}=0.01$ is $v_{s}=0.6754$. The coordination number is $\mathscr{C} \approx 7.16$ in this state. After stress-relaxation, these values have changed to $v \approx 0.629$ and $\mathscr{C} \approx 6.19$. A different preparation procedure (with adhesion $k_{c} / k_{2}=0$ during sintering) does not lead to a difference in density after sintering. However, one observes $v \approx 0.630$ and $\mathscr{C} \approx 6.23$ after relaxation. For both preparation procedures the tension test results are virtually identical, so that only the first procedure is used in the following.

\begin{tabular}{|l||l|l|l|}
\hline Symbol & Value & rescaled units & SI-units \\
\hline \hline$t_{u}$ & 1 & $1 \mu \mathrm{s}$ & $10^{-6} \mathrm{~s}$ \\
$x_{u}$ & 1 & $1 \mathrm{~mm}$ & $10^{-3} \mathrm{~m}$ \\
$m_{u}$ & 1 & $1 \mathrm{mg}$ & $10^{-6} \mathrm{~kg}$ \\
\hline$a_{0}$ & 0.005 & $5 \mu \mathrm{m}$ & $5.10^{-6} \mathrm{~m}$ \\
$\rho$ & 2 & $2 \mathrm{mg} / \mathrm{mm}^{3}$ & $2000 \mathrm{~kg} / \mathrm{m}^{3}$ \\
\hline$k=k_{2}$ & 5 & $5 \mathrm{mg} / \mu \mathrm{s}^{2}$ & $5.10^{6} \mathrm{~kg} / \mathrm{s}^{2}$ \\
$k_{1} / k$ & 0.5 & & \\
$k_{c} / k$ & 0.5 & & \\
$k_{t} / k$ & 0.2 & & \\
$k_{r} / k=k_{o} / k$ & 0.1 & & \\
\hline$\phi_{f}$ & 0.05 & & \\
\hline$\mu=\mu_{d}=\mu_{s}$ & 1 & & \\
$\phi_{d}=\mu_{d} / \mu_{s}$ & 1 & & \\
$\mu_{r}=\mu_{o}$ & 0.1 & & \\
\hline$\gamma=\gamma_{n}$ & $5.10^{-5}$ & $5.10^{-5} \mathrm{mg} / \mu \mathrm{s}$ & $5.10^{1} \mathrm{~kg} / \mathrm{s}$ \\
$\gamma_{t} / \gamma$ & 0.2 & & \\
$\gamma_{r} / \gamma=\gamma_{o} / \gamma$ & 0.05 & & \\
$\gamma_{b} / \gamma$ & 4.0 & & \\
$\gamma_{b r} / \gamma$ & 1.0 & & \\
\hline
\end{tabular}

Table 2 Microscopic material parameters used (second column), if not explicitly specified. The third column contains these values in the appropriate units, i.e., when the time-, length-, and massunit are $\mu \mathrm{s}, \mathrm{mm}$, and $\mathrm{mg}$, respectively. Column four contains the parameters in SI-units. Energy, force, acceleration, and stress have to be scaled with factors of $1,10^{3}, 10^{9}$, and $10^{9}$, respectively, for a transition from reduced to SI-units.

The material parameters used for the particle contacts are given in table 2 . The particle-wall contact parameters are the same, except for cohesion and friction, for which $k_{c}^{\text {wall }} / k_{2}=20$ and $\mu^{\text {wall }}=10$ are used - the former during all stages, the latter only during tensile testing.

The choice of numbers and units is such that the particles correspond spheres with several microns in radius. The magnitude of stiffness $k$ cannot be compared directly with the material bulk modulus $C$, since it is a contact property. However, there are relations from micro-macro transition analysis, which allow to relate $k$ and $C \sim k \mathscr{C} a^{2} / V[35,39]$.

Using the parameter $k=k_{2}$ in Eq. (4) leads to a typical contact duration (halfperiod) $t_{c} \approx 6.510^{-4} \mu \mathrm{s}$, for a normal collision of a large and a small particle with 
$\gamma=0$. Accordingly, an integration time-step of $t_{\mathrm{MD}}=5 \cdot 10^{-6} \mu \mathrm{s}$ is used, in order to allow for a "safe" integration of the equations of motion. Note that not only the normal "eigenfrequency" but also the eigenfrequencies in tangential and rotational direction have to be considered as well as the viscous response times $t_{\gamma} \approx m / \gamma$. All of the physical time-scales should be considerably larger than $t_{\mathrm{MD}}$, whereas the viscous response times should be even larger, so that $t_{\gamma}>t_{c}>t_{\mathrm{MD}}$. A more detailed discussion of all the effects due to the interplay between the model parameters and the related times is, however, far from the scope of this paper.

\subsubsection{Compressive and tensile strength}

The tensile (compressive) test is performed uni-axially in $x$-direction by increasing (reducing) slowly and smoothly the distance between the two sticky walls. (The same initial sample, prepared with $k_{c} / k_{2}=1 / 2$, is used for all tests reported here.)

The stress-strain curves for different cohesion are plotted in Fig. 2, for both tension and compression. Note that the shape of the curves and the apparent material behavior (ductile, quasi-brittle, and brittle) depends not only on the contact parameters, but also on the rate the deformation is performed (due to the viscous forces introduced above). The present data are for moderate to slow deformation. Faster deformation leads to even smoother curves with larger apparent strength, while considerably slower deformation leads to more brittle behavior (with sharper drops of stress) and somewhat smaller strength.
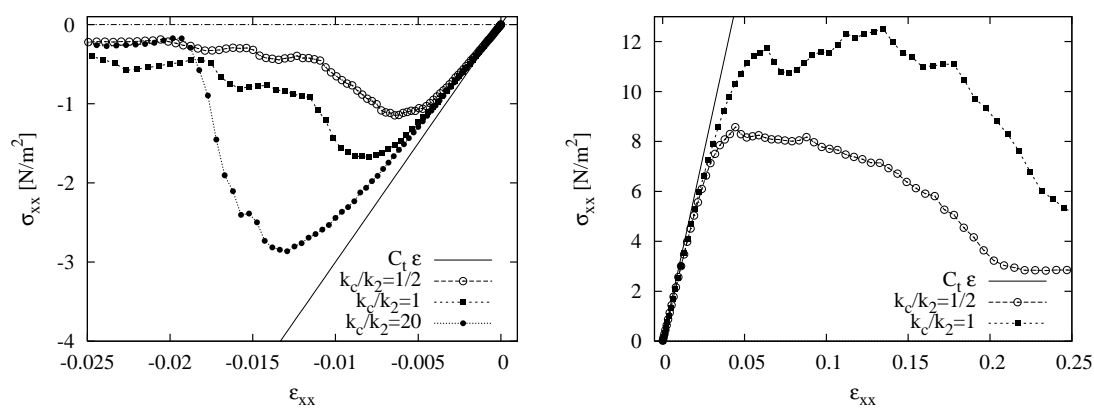

Fig. 2 (Left) Axial tensile stress plotted against tensile strain for simulations with weak, moderate and strong particle contact adhesion; the $k_{c} / k_{2}$ values are given in the inset. The line gives a fit to the linear elastic regime with $C_{t}=3.10^{11} \mathrm{~N} / \mathrm{m}^{2}$. (Right) Axial compressive stress plotted against compressive strain for two of the parameter sets from the top panel. The initial slope is the same as in the top panel, indicating that the linear elastic regime is identical for tension and compression.

The axial tensile stress initially increases linearly with strain, practically independent from the contact adhesion strength. With increasing strain, a considerable number of contacts are opened due to tension - contacts open more easily for smaller 
adhesion (data not shown). This leads to a decrease of the stress-strain slope, then the stress reaches a maximum and, for larger strain, turns into a softening failure mode. As expected, the maximal stress is increasing with contact adhesion $k_{c} / k_{2}$. The compressive strength is $6-7$ times larger than the tensile strength, and a larger adhesion force also allows for larger deformation before failure. The sample with weakest adhesion, $k_{c} / k_{2}=1 / 2$, shows tensile and compressive failure at strains $\varepsilon_{x x} \approx-0.006$ and $\varepsilon_{x x} \approx 0.045$, respectively.

Note that for tension, the post-peak behavior for the test with $k_{c} / k_{2}=20$ is different from the other two cases, due to the strong particle-particle contact adhesion. In this case, the tensile fracture occurs at the wall (except for a few particles that remain in contact with the wall). This is in contrast to the other cases with smaller bulk-adhesion, where the fracture occurs in the bulk, see Fig. 3.
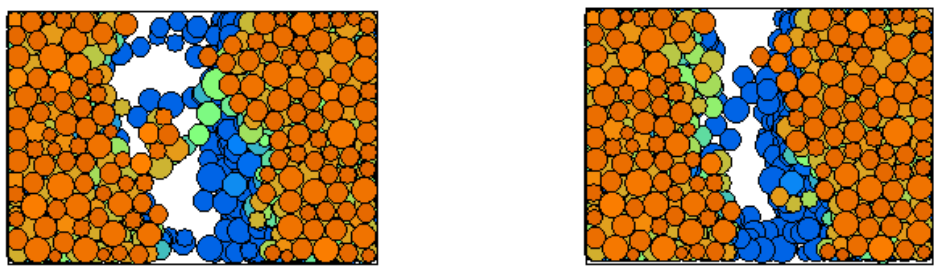

Fig. 3 Snapshots from tensile tests with $k_{c} / k_{2}=1 / 5$ and 1 , at horizontal strain of $\varepsilon_{x x} \approx-0.8$. The color code denotes the distance from the viewer: blue, green, and red correspond to large, moderate, and short distance.

\section{Hard Sphere Molecular Dynamics}

In this section, the hard sphere model is introduced together with the event-driven algorithm. A generalized model takes into account the finite contact duration of realistic particles and, besides providing a physcial parameter, saves computing time because it avoids the "inelastic collapse".

In the framework of the hard sphere model, particles are assumed to be perfectly rigid and they follow an undisturbed motion until a collision occurs as described below. Due to the rigidity of the interaction, the collisions occur instantaneously, so that an event-driven simulation method $[28,51,57,56,55]$ can be used. Note that the ED method was only recently implemented in parallel [29, 57]; however, we avoid to discuss this issue in detail. 
The instantaneous nature of hard sphere collisions is artificial, however, it is a valid limit in many circumstances. Even though details of the contact- or collision behavior of two particles are ignored, the hard sphere model is valid when binary collisions dominate and multi-particle contacts are rare [44]. The lack of physical information in the model allows a much simpler treatment of collisions than described in section 2 by just using a collision matrix based on momentum conservation and energy loss rules. For the sake of simplicity, we restrict ourselves to smooth hard spheres here. Collision rules for rough spheres are extensively discussed elsewhere, see e.g. [47, 18], and references therein.

\subsection{Smooth Hard Sphere Collision Model}

Between collisions, hard spheres fly independently from each other. A change in velocity - and thus a change in energy - can occur only at a collision. The standard interaction model for instantaneous collisions of identical particles with radius $a$, and mass $m$, is used in the following. The post-collisional velocities $v^{\prime}$ of two collision partners in their center of mass reference frame are given, in terms of the pre-collisional velocities $v$, by

$$
v_{1,2}^{\prime}=v_{1,2} \mp(1+r) v_{n} / 2,
$$

with $v_{n} \equiv\left[\left(v_{1}-v_{2}\right) \cdot n\right] n$, the normal component of the relative velocity $v_{1}-v_{2}$, parallel to $n$, the unit vector pointing along the line connecting the centers of the colliding particles. If two particles collide, their velocities are changed according to Eq. (24), with the change of the translational energy at a collision $\Delta E=-m_{12}(1-$ $\left.r^{2}\right) v_{n}^{2} / 2$, with dissipation for restitution coefficients $r<1$.

\subsection{Event-Driven Algorithm}

Since we are interested in the behavior of granular particles, possibly evolving over several decades in time, we use an event-driven (ED) method which discretizes the sequence of events with a variable time step adapted to the problem. This is different from classical DEM simulations, where the time step is usually fixed.

In the ED simulations, the particles follow an undisturbed translational motion until an event occurs. An event is either the collision of two particles or the collision of one particle with a boundary of a cell (in the linked-cell structure) [5]. The cells have no effect on the particle motion here; they were solely introduced to accelerate the search for future collision partners in the algorithm.

Simple ED algorithms update the whole system after each event, a method which is straightforward, but inefficient for large numbers of particles. In Ref. [28] an ED algorithm was introduced which updates only those two particles involved in the 
last collision. Because this algorithm is "asynchronous" in so far that an event, i.e. the next event, can occur anywhere in the system, it is so complicated to parallelize it [57]. For the serial algorithm, a double buffering data structure is implemented, which contains the 'old' status and the 'new' status, each consisting of: time of event, positions, velocities, and event partners. When a collision occurs, the 'old' and 'new' status of the participating particles are exchanged. Thus, the former 'new' status becomes the actual 'old' one, while the former 'old' status becomes the 'new' one and is then free for the calculation and storage of possible future events. This seemingly complicated exchange of information is carried out extremely simply and fast by only exchanging the pointers to the 'new' and 'old' status respectively. Note that the 'old' status of particle $i$ has to be kept in memory, in order to update the time of the next contact, $t_{i j}$, of particle $i$ with any other object $j$ if the latter, independently, changed its status due to a collision with yet another particle. During the simulation such updates may be neccessary several times so that the predicted 'new' status has to be modified.

The minimum of all $t_{i j}$ is stored in the 'new' status of particle $i$, together with the corresponding partner $j$. Depending on the implementation, positions and velocities after the collision can also be calculated. This would be a waste of computer time, since before the time $t_{i j}$, the predicted partners $i$ and $j$ might be involved in several collisions with other particles, so that we apply a delayed update scheme [28]. The minimum times of event, i.e. the times, which indicate the next event for a certain particle, are stored in an ordered heap tree, such that the next event is found at the top of the heap with a computational effort of $O(1)$; changing the position of one particle in the tree from the top to a new position needs $O(\log N)$ operations. The search for possible collision partners is accelerated by the use of a standard linked-cell data structure and consumes $O(1)$ of numerical resources per particle. In total, this results in a numerical effort of $O(N \log N)$ for $N$ particles. For a detailed description of the algorithm see Ref. [28]. Using all these algorithmic tricks, we are able to simulate about $10^{5}$ particles within reasonable time on a low-end PC [45], where the particle number is more limited by memory than by CPU power. Parallelization, however, is a means to overcome the limits of one processor [57].

As a final remark concerning ED, one should note that the disadvantages conncected to the assumptions made that allow to use an event driven algorithm limit the applicability of this method. Within their range of applicability, ED simulations are typically much faster than DEM simulations, since the former accounts for a collision in one basic operation (collision matrix), whereas the latter requires about one hundred basic steps (integration time steps). Note that this statement is also true in the dense regime. In the dilute regime, both methods give equivalent results, because collisions are mostly binary [41]. When the system becomes denser, multiparticle collisions can occur and the rigidity assumption within the ED hard sphere approach becomes invalid.

The most striking difference between hard and soft spheres is the fact that soft particles dissipate less energy when they are in contact with many others of their kind. In the following chapter, the so called TC model is discussed as a means to account for the contact duration $t_{c}$ in the hard sphere model. 


\section{The Link between ED and DEM via the TC Model}

In the ED method the contact duration is implicitly zero, matching well the corresponding assumption of instantaneous contacts used for the kinetic theory $[17,22]$. Due to this artificial simplification (which disregards the fact that a real contact takes always finite time) ED algorithms run into problems when the time between events $t_{\mathrm{n}}$ gets too small: In dense systems with strong dissipation, $t_{\mathrm{n}}$ may even tend towards zero. As a consequence the so-called "inelastic collapse" can occur, i.e. the divergence of the number of events per unit time. The problem of the inelastic collapse [54] can be avoided using restitution coefficients dependent on the time elapsed since the last event $[51,44]$. For the contact that occurs at time $t_{i j}$ between particles $i$ and $j$, one uses $r=1$ if at least one of the partners involved had a collision with another particle later than $t_{i j}-t_{\mathrm{c}}$. The time $t_{\mathrm{c}}$ can be seen as a typical duration of a contact, and allows for the definition of the dimensionless ratio

$$
\tau_{c}=t_{c} / t_{n}
$$

The effect of $t_{\mathrm{c}}$ on the simulation results is negligible for large $r$ and small $t_{\mathrm{c}}$; for a more detailed discussion see $[51,45,44]$.

In assemblies of soft particles, multi-particle contacts are possible and the inelastic collapse is avoided. The TC model can be seen as a means to allow for multi-particle collisions in dense systems [43, 30, 51]. In the case of a homogeneous cooling system (HCS), one can explicitly compute the corrected cooling rate (r.h.s.) in the energy balance equation

$$
\frac{d}{d \tau} E=-2 I\left(E, t_{c}\right)
$$

with the dimensionless time $\tau=(2 / 3) A t / t_{E}(0)$ for $3 \mathrm{D}$ systems, scaled by $A=(1-$ $\left.r^{2}\right) / 4$, and the collision rate $t_{E}^{-1}=(12 / a) v g(v) \sqrt{T /(\pi m)}$, with $T=2 K /(3 N)$. In these units, the energy dissipation rate $I$ is a function of the dimensionless energy $E=K / K(0)$ with the kinetic energy $K$, and the cut-off time $t_{c}$. In this representation, the restitution coefficient is hidden in the rescaled time via $A=A(r)$, so that inelastic hard sphere simulations with different $r$ scale on the same master-curve. When the classical dissipation rate $E^{3 / 2}[17]$ is extracted from $I$, so that $I\left(E, t_{c}\right)=J\left(E, t_{c}\right) E^{3 / 2}$, one has the correction-function $J \rightarrow 1$ for $t_{c} \rightarrow 0$. The deviation from the classical HCS is [44]:

$$
J\left(E, t_{c}\right)=\exp (\Psi(x)),
$$

with the series expansion $\Psi(x)=-1.268 x+0.01682 x^{2}-0.0005783 x^{3}+\mathscr{O}\left(x^{4}\right)$ in the collision integral, with $x=\sqrt{\pi} t_{c} t_{E}^{-1}(0) \sqrt{E}=\sqrt{\pi} \tau_{c}(0) \sqrt{E}=\sqrt{\pi} \tau_{c}$ [44]. This is close to the result $\Psi_{\mathrm{LM}}=-2 x / \sqrt{\pi}$, proposed by Luding and McNamara, based on probabilistic mean-field arguments [51] ${ }^{3}$.

Given the differential equation (26) and the correction due to multi-particle contacts from Eq. (27), it is possible to obtain the solution numerically, and to compare

\footnotetext{
${ }^{3} \Psi_{\mathrm{LM}}$ thus neglects non-linear terms and underestimates the linear part
} 

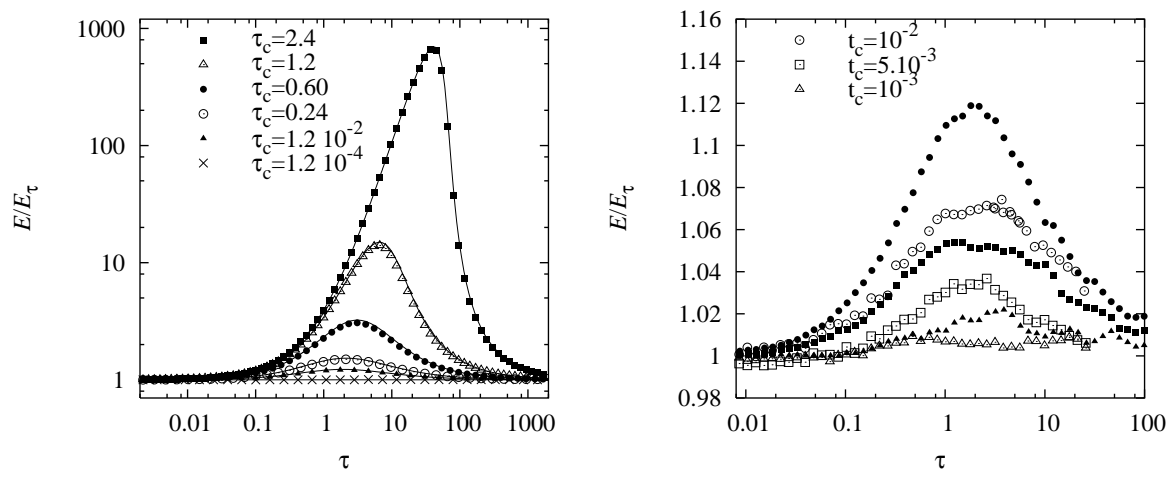

Fig. 4 (Left) Deviation from the HCS, i.e. rescaled energy $E / E_{\tau}$, where $E_{\tau}$ is the classical solution $E_{\tau}=(1+\tau)^{-2}$. The data are plotted against $\tau$ for simulations with different $\tau_{c}(0)=t_{c} / t_{E}(0)$ as given in the inset, with $r=0.99$, and $N=8000$. Symbols are ED simulation results, the solid line results from the third order correction. (Right) $E / E_{\tau}$ plotted against $\tau$ for simulations with $r=0.99$, and $N=2197$. Solid symbols are ED simulations, open symbols are DEM (soft particle simulations) with three different $t_{c}$ as given in the inset.

it to the classical $E_{\tau}=(1+\tau)^{-2}$ solution. Simulation results are compared to the theory in Fig. 4 (left). The agreement between simulations and theory is almost perfect in the examined range of $t_{c}$-values, only when deviations from homogeneity are evidenced one expects disagreement between simulation and theory. The fixed cut-off time $t_{c}$ has no effect when the time between collisions is very large $t_{E} \gg t_{c}$, but strongly reduces dissipation when the collisions occur with high frequency $t_{E}^{-1} \gtrsim t_{c}^{-1}$. Thus, in the homogeneous cooling state, there is a strong effect initially, and if $t_{c}$ is large, but the long time behavior tends towards the classical decay $E \rightarrow E_{\tau} \propto \tau^{-2}$.

The final check if the ED results obtained using the TC model are reasonable is to compare them to DEM simulations, see Fig. 4 (right). Open and solid symbols correspond to soft and hard sphere simulations respectively. The qualitative behavior (the deviation from the classical HCS solution) is identical: The energy decay is delayed due to multi-particle collisions, but later the classical solution is recovered. A quantitative comparison shows that the deviation of $E$ from $E_{\tau}$ is larger for ED than for DEM, given that the same $t_{c}$ is used. This weaker dissipation can be understood from the strict rule used for ED: Dissipation is inactive if any particle had a contact already. The disagreement between ED and DEM is systematic and should disappear if an about 30 per-cent smaller $t_{c}$ value is used for ED. The disagreement is also plausible, since the TC model disregards all dissipation for multi-particle contacts, while the soft particles still dissipate energy - even though much less - in the case of multi-particle contacts.

The above simulations show that the TC model is in fact a "trick" to make hard particles soft and thus connecting between the two types of simulation models: soft and hard. The only change made to traditional ED involves a reduced dissipation for (rapid) multi-particle contacts. 


\section{The Stress in Particle Simulations}

The stress tensor is a macroscopic quantity that can be obtained by measurement of forces per area, or via a so-called micro-macro homogenization procedure. Both methods will be discussed below. During derivation, it also turns out that stress has two contributions, the first is the "static stress" due to particle contacts, a potential energy density, the second is the "dynamics stress" due to momentum flux, like in the ideal gas, a kinetic energy density. For the sake of simplicity, we restrict ourselves to the case of smooth spheres here.

\subsection{Dynamic Stress}

For dynamic systems, one has momentum transport via flux of the particles. This simplest contribution to the stress tensor is the standard stress in an ideal gas, where the atoms (mass points) move with a certain fluctuation velocity $v_{i}$. The kinetic energy $E=\sum_{i=1}^{N} m v_{i}^{2} / 2$ due to the fluctuation velocity $v_{i}$ can be used to define the temperature of the gas $k_{B} T=2 E /(\mathscr{D} N)$, with the dimension $\mathscr{D}$ and the particle number $N$. Given a number density $n=N / V$, the stress in the ideal gas is then isotropic and thus quantified by the pressure $p=n k_{B} T$; note that we will disregard $k_{B}$ in the following. In the general case, the dynamic stress is $\sigma=(1 / V) \sum_{i} m_{i} v_{i} \otimes v_{i}$, with the dyadic tensor product denoted by ' $\otimes$ ', and the pressure $p=\operatorname{tr} \sigma / \mathscr{D}=n T$ is the kinetic energy density.

The additional contribution to the stress is due to collisions and contacts and will be derived from the principle of virtual displacement for soft interaction potentials below, and then be modified for hard sphere systems.

\subsection{Static Stress from Virtual Displacements}

From the centers of mass $r_{1}$ and $r_{2}$ of two particles, we define the so-called branch vector $l=r_{1}-r_{2}$, with the reference distance $l=|l|=2 a$ at contact, and the corresponding unit vector $n=l / l$. The deformation in the normal direction, relative to the reference configuration, is defined as $\delta=2 a n-l$. A virtual change of the deformation is then

$$
\partial \delta=\delta^{\prime}-\delta \approx \partial l=\varepsilon \cdot l,
$$

where the prime denotes the deformation after the virtual displacement described by the tensor $\varepsilon$. The corresponding potential energy density due to the contacts of one pair of particles is $u=k \delta^{2} /(2 V)$, expanded to second order in $\delta$, leading to the virtual change

$$
\partial u=\frac{k}{V}\left(\delta \cdot \partial \delta+\frac{1}{2}(\partial \delta)^{2}\right) \approx \frac{k}{V} \delta \cdot \partial l^{n},
$$


where $k$ is the spring stiffness (the prefactor of the quadratic term in the series expansion of the interaction potential), $V$ is the averaging volume, and $\partial l^{n}=n(n \cdot \varepsilon \cdot l)$ is the normal component of $\partial l$. Note that $\partial u$ depends only on the normal component of $\partial \delta$ due to the scalar product with $\delta$, which is parallel to $n$.

From the potential energy density, we obtain the stress from a virtual deformation by differentiation with respect to the deformation tensor components

$$
\sigma=\frac{\partial u}{\partial \varepsilon}=\frac{k}{V} \delta \otimes l=\frac{1}{V} f \otimes l
$$

where $f=k \delta$ is the force acting at the contact, and the dyadic product $\otimes$ of two vectors leads to a tensor of rank two.

\subsection{Stress for Soft and Hard Spheres}

Combining the dynamic and the static contributions to the stress tensor [49], one has for smooth, soft spheres:

$$
\sigma=\frac{1}{V}\left[\sum_{i} m_{i} v_{i} \otimes v_{i}-\sum_{c \in V} f_{c} \otimes l_{c}\right],
$$

where the right sum runs over all contacts $c$ in the averaging volume $V$. Replacing the force vector by momentum change per unit time, one obtains for hard spheres:

$$
\sigma=\frac{1}{V}\left[\sum_{i} m_{i} v_{i} \otimes v_{i}-\frac{1}{\Delta t} \sum_{n} \sum_{j} p_{j} \otimes l_{j}\right],
$$

where $p_{j}$ and $l_{j}$ are the momentum change and the center-contact vector of particle $j$ at collision $n$, respectively. The sum in the left term runs over all particles $i$, the first sum in the right term runs over all collisions $n$ occurring in the averaging time $\Delta t$, and the second sum in the right term concerns the collision partners of collision $n$ [51].

Exemplary stress computations from DEM and ED simulations are presented in the following section.

\section{2D Simulation Results}

Stress computations from two dimensional DEM and ED simulations are presented in the following subsections. First, a global equation of state, valid for all densities, is proposed based on ED simulations, and second, the stress tensor from a slow, 
quasi-static deformation is computed from DEM simulations with frictional particles.

\subsection{The Equation of State from $\mathrm{ED}$}

The mean pressure in two dimensions is $p=\left(\sigma_{1}+\sigma_{2}\right) / 2$, with the eigenvalues $\sigma_{1}$ and $\sigma_{2}$ of the stress tensor $[48,49,32]$. The 2D dimensionless, reduced pressure $P=p /(n T)-1=p V / E-1$ contains only the collisional contribution and the simulations agree nicely with the theoretical prediction $P_{2}=2 v g_{2}(v)$ for elastic systems, with the pair-correlation function $g_{2}(v)=(1-7 v / 16) /(1-v)^{2}$, and the volume fraction $v=N \pi a^{2} / V$, see Fig. 5. A better pair-correlation function is

$$
g_{4}(v)=\frac{1-7 v / 16}{(1-v)^{2}}-\frac{v^{3} / 16}{8(1-v)^{4}},
$$

which defines the non-dimensional collisional stress $P_{4}=2 v g_{4}(v)$. For a system with homogeneous temperature, as a remark, the collision rate is proportional to the dimensionless pressure $t_{n}^{-1} \propto P$.

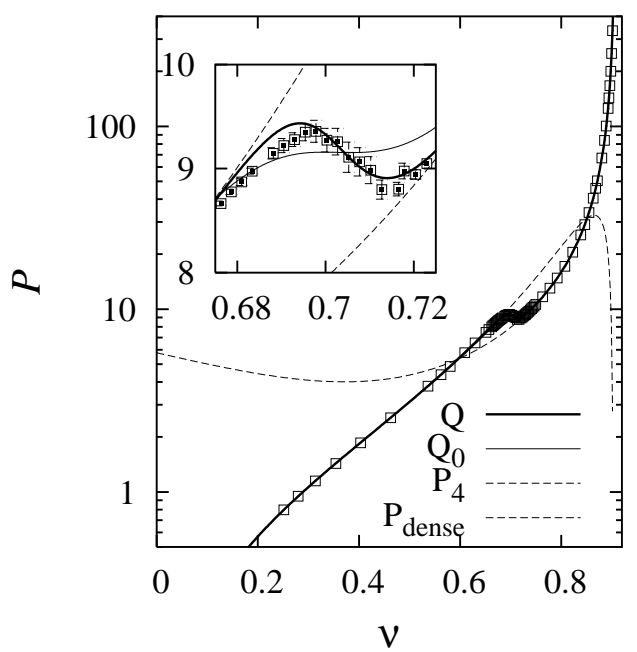

Fig. 5 The dashed lines are $P_{4}$ and $P_{\text {dense }}$ as functions of the volume fraction $v$, and the symbols are simulation data, with standard deviations as given by the error bars in the inset. The thick solid line is $Q$, the corrected global equation of state from Eq. (34), and the thin solid line is $Q_{0}$ without empirical corrections.

When plotting $P$ against $v$ with a logarithmic vertical axis, in Fig. 5, the simulation results can almost not be distinguished from $P_{2}$ for $v<0.65$, but $P_{4}$ leads 
to better agreement up to $v=0.67$. Crystallization is evidenced at the point of the liquid-solid transition $v_{c} \approx 0.7$, and the data clearly deviate from $P_{4}$. The pressure is strongly reduced due to the increase of free volume caused by ordering. Eventually, the data diverge at the maximum packing fraction $v_{\max }=\pi /(2 \sqrt{3})$ for a perfect triangular array.

For high densities, one can compute from free-volume models, the reduced pressure $P_{\mathrm{fv}}=2 v_{\max } /\left(v_{\max }-v\right)$. Slightly different functional forms do not lead to much better agreement [32]. Based on the numerical data, we propose the corrected high density pressure $P_{\text {dense }}=P_{\mathrm{fv}} h\left(v_{\max }-v\right)-1$, with the empirical fit function $h(x)=1+c_{1} x+c_{3} x^{3}$, and $c_{1}=-0.04$ and $c_{3}=3.25$, in perfect agreement with the simulation results for $v \geq 0.73$.

Since, to our knowledge, there is no conclusive theory available to combine the disordered and the ordered regime [23], we propose a global equation of state

$$
Q=P_{4}+m(v)\left[P_{\text {dense }}-P_{4}\right],
$$

with an empirical merging function $m(v)=\left[1+\exp \left(-\left(v-v_{c}\right) / m_{0}\right)\right]^{-1}$, which selects $P_{4}$ for $v \ll v_{c}$ and $P_{\text {dense }}$ for $v \gg v_{c}$, with the transition density $v_{c}$ and the width of the transition $m_{0}$. In Fig. 5, the fit parameters $v_{c}=0.702$ and $m_{0} \approx 0.0062$ lead to qualitative and quantitative agreement between $Q$ (thick line) and the simulation results (symbols). However, a simpler version $Q_{0}=P_{2}+m(v)\left[P_{\mathrm{fv}}-P_{2}\right]$, (thin line) without empirical corrections leads already to reasonable agreement when $v_{c}=0.698$ and $m_{0}=0.0125$ are used. In the transition region, this function $Q_{0}$ has no negative slope but is continuous and differentiable, so that it allows for an easy and compact numerical integration of $P$. We selected the parameters for $Q_{0}$ as a compromise between the quality of the fit on the one hand and the simplicity and treatability of the function on the other hand.

As an application of the global equation of state, the density profile of a dense granular gas in the gravitational field has been computed for monodisperse [49] and bidisperse situations $[48,32]$. In the latter case, however, segregation was observed and the mixture theory could not be applied. The equation of state and also other transport properties are extensively discussed in Refs. [4, 1, 3, 2] for 2D, bi-disperse systems.

\subsection{Quasi-static DEM simulations}

In contrast to the dynamic, collisional situation discussed in the previous section, a quasi-static situation, with all particles almost at rest most of the time, is discussed in the following. 


\subsubsection{Model Parameters}

The systems examined in the following contain $N=1950$ particles with radii $a_{i}$ randomly drawn from a homogeneous distribution with minimum $a_{\min }=0.510^{-3} \mathrm{~m}$ and maximum $a_{\max }=1.510^{-3} \mathrm{~m}$. The masses $m_{i}=(4 / 3) \rho \pi a_{i}^{3}$, with the density $\rho=2.010^{3} \mathrm{~kg} \mathrm{~m}^{-3}$, are computed as if the particles were spheres. This is an artificial choice and introduces some dispersity in mass in addition to the dispersity in size. Since we are mainly concerned about slow deformation and equilibrium situations, the choice for the calculation of mass should not matter. The total mass of the particles in the system is thus $M \approx 0.02 \mathrm{~kg}$ with the typical reduced mass of a pair of particles with mean radius, $m_{12} \approx 0.4210^{-5} \mathrm{~kg}$. If not explicitly mentioned, the material parameters are $k_{2}=10^{5} \mathrm{~N} \mathrm{~m}^{-1}$ and $\gamma_{0}=0.1 \mathrm{~kg} \mathrm{~s}^{-1}$. The other springconstants $k_{1}$ and $k_{c}$ will be defined in units of $k_{2}$. In order to switch on adhesion, $k_{1}<k_{2}$ and $k_{c}>0$ is used; if not mentioned explicitly, $k_{1}=k_{2} / 2$ is used, and $k_{2}$ is constant, independent of the maximum overlap previously achieved.

Using the parameters $k_{1}=k_{2}$ and $k_{c}=0$ in Eq. (4) leads to a typical contact duration (half-period): $t_{c} \approx 2.0310^{-5} \mathrm{~s}$ for $\gamma_{0}=0, t_{c} \approx 2.0410^{-5} \mathrm{~s}$ for $\gamma_{0}=0.1 \mathrm{~kg} \mathrm{~s}^{-1}$, and $t_{c} \approx 2.2110^{-5} \mathrm{~s}$ for $\gamma_{0}=0.5 \mathrm{~kg} \mathrm{~s}^{-1}$ for a collision. Accordingly, an integration time-step of $t_{\mathrm{DEM}}=510^{-7} \mathrm{~s}$ is used, in order to allow for a 'safe' integration of contacts involving smaller particles. Large values of $k_{c}$ lead to strong adhesive forces, so that also more energy can be dissipated in one collision. The typical response time of the particle pairs, however, is not affected so that the numerical integration works well from a stability and accuracy point of view.

\subsubsection{Boundary Conditions}

The experiment chosen is the bi-axial box set-up, see Fig. 6, where the left and bottom walls are fixed, and stress- or strain-controlled deformation is applied. In the first case a wall is subject to a predefined pressure, in the second case, the wall is subject to a pre-defined strain. In a typical 'experiment', the top wall is strain controlled and slowly shifted downwards while the right wall moves stress controlled, dependent on the forces exerted on it by the material in the box. The strain-controlled position of the top wall as function of time $t$ is here

$$
z(t)=z_{\mathrm{f}}+\frac{z_{0}-z_{\mathrm{f}}}{2}(1+\cos \omega t), \text { with } \varepsilon_{\mathrm{zz}}=1-\frac{z}{z_{0}},
$$

where the initial and the final positions $z_{0}$ and $z_{\mathrm{f}}$ can be specified together with the rate of deformation $\omega=2 \pi f$ so that after a half-period $T / 2=1 /(2 f)$ the extremal deformation is reached. With other words, the cosine is active for $0 \leq \omega t \leq \pi$. For larger times, the top-wall is fixed and the system can relax indefinitely. The cosine function is chosen in order to allow for a smooth start-up and finish of the motion so that shocks and inertia effects are reduced, however, the shape of the function is arbitrary as long as it is smooth.

The stress-controlled motion of the side-wall is described by 

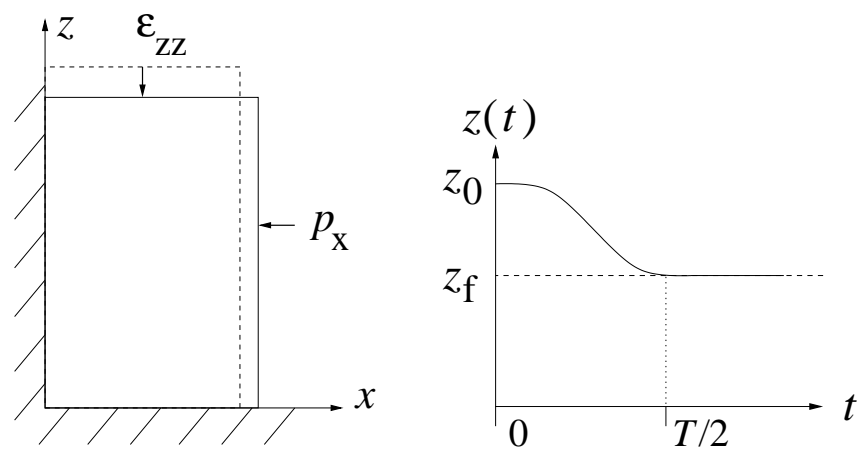

Fig. 6 (Left) Schematic drawing of the model system. (Right) Position of the top-wall as function of time for the strain-controlled situation.

$$
m_{\mathrm{w}} \ddot{x}(t)=F_{\mathrm{X}}(t)-p_{\mathrm{x}} z(t)-\gamma_{\mathrm{w}} \dot{x}(t),
$$

where $m_{\mathrm{w}}$ is the mass of the right side wall. Large values of $m_{\mathrm{W}}$ lead to slow adaption, small values allow for a rapid adaption to the actual situation. Three forces are active: (i) the force $F_{\mathrm{X}}(t)$ due to the bulk material, (ii) the force $-p_{\mathrm{X}} z(t)$ due to the external pressure, and (iii) a strong frictional force which damps the motion of the wall so that oscillations are reduced.

\subsubsection{Initial Configuration and Compression}

Initially, the particles are randomly distributed in a huge box, with rather low overall density. Then the box is compressed, either by moving the walls to their desired position, or by defining an external pressure $p=p_{\mathrm{x}}=p_{\mathrm{z}}$, in order to achieve an isotropic initial condition. Starting from a relaxed, isotropic initial configuration, the strain is applied to the top wall and the response of the system is examined. In Fig. 7, snapshots from a typical simulation are shown during compression.

In the following, simulations are presented with different side pressures $p=20$, $40,100,200,400$, and 500. The behavior of the averaged scalar and tensor variables during the simulations is examined in more detail for situations with small and large confining pressure. The averages are performed such that ten to twenty per-cent of the total volume are disregarded in the vicinity of each wall in order to avoid boundary effects. A particle contact is taken into account for the average if the contact point lies within the averaging volume $V$.

\subsubsection{Compression and Dilation}

The first quantity of interest is the density (volume fraction) $v$ and, related to it, the volumetric strain $\varepsilon_{V}=\Delta V / V$. From the averaged data, we evidence compression 
$\varepsilon_{z z}=0$

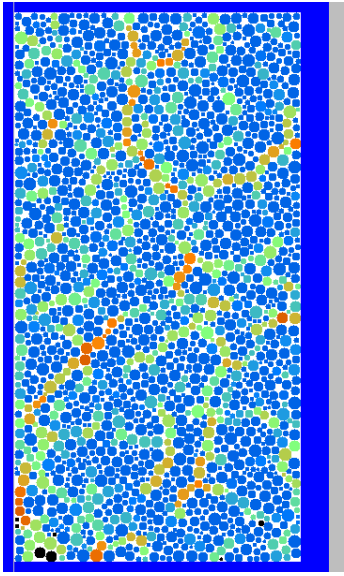

$\varepsilon_{z z}=0.042$

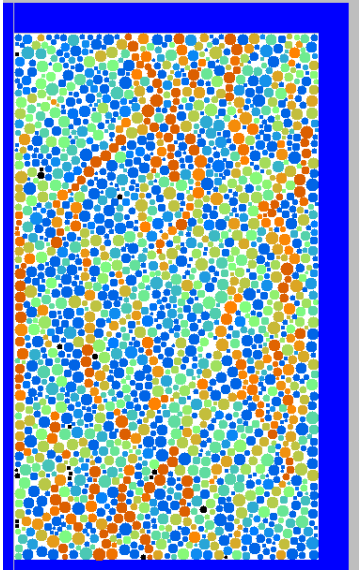

$\varepsilon_{z z}=0.091$

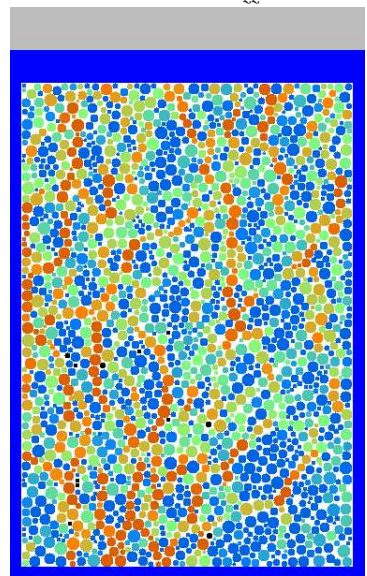

Fig. 7 Snapshots of the simulation at different $\varepsilon_{z z}$ for constant side pressure $p$. The color code corresponds to the potential energy of each particle, decaying from red over green to blue and black. The latter black particles are so-called rattlers that do not contribute to the static contact network.

for small deformation and large side pressure. This initial regime follows strong dilation, for all pressures, until a quasi-steady-state is reached, where the density is almost constant besides a weak tendency towards further dilation.
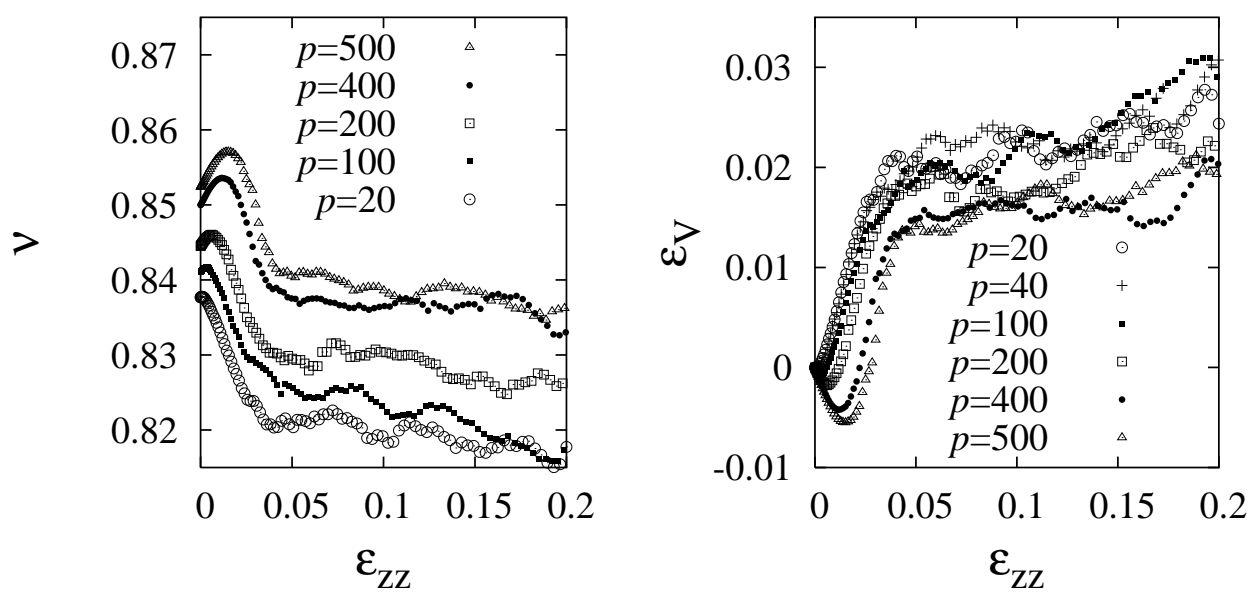

Fig. 8 (Left) Volume fraction $v=\sum_{i} \pi a_{i}^{2} / V$ for different confining pressure $p$. (Right) Volumetric strain - negative values mean compression, whereas positive values correspond to dilation. 
An initially dilute granular medium (weak confining pressure) thus shows dilation from the beginning, whereas a denser granular material (strong confining pressure) can be compressed even further by the relatively strong external forces until dilation starts. The range of density changes is about 0.02 in volume fraction and spans up to $3 \%$ changes in volumetric strain.

From the initial slope, one can obtain the Poisson ratio of the bulk material, and from the slope in the dilatant regime, one obtains the so-called dilatancy angle, a measure of the magnitude of dilatancy required before shear is possible [46, 33].

The anisotropy of the granular packing is quantified by the deviatoric fabric (data not shown). The anisotropy is initially of the order of a few percent at most - thus the initial configurations are already not perfectly isotropic - even though isotropically prepared. With increasing deviatoric deformation, the anisotropy grows, reaches a maximum and then saturates on a lower level in the critical state flow regime. The scaled fabric grows faster for smaller side pressure and is also relatively larger for smaller $p$. The non-scaled fabric deviator, astonishingly, grows to values around $f_{D}^{\max } \operatorname{tr} F \approx 0.56 \pm 0.03$, independently of the side pressures used here (data not shown, see [33, 34] for details). Using the definition $f_{D}:=\operatorname{dev} F / \operatorname{tr} F$, the functional behavior,

$$
\frac{\partial f_{D}}{\partial \varepsilon_{D}}=\beta_{f}\left(f_{D}^{\max }-f_{D}\right)
$$

was evidenced from simulations in Ref. [33], with $f_{D}^{\max } \operatorname{tr} F \approx$ const., and the deviatoric rate of approach $\beta_{f}=\beta_{f}(p)$, decreasing with increasing side pressure. The differential equation is solved by an exponential function that describes the approach of the anisotropy $f_{D}$ to its maximal value, $1-\left(f_{D} / f_{D}^{\max }\right)=\exp \left(-\beta_{f} \varepsilon_{D}\right)$, but not beyond.

\subsubsection{Stress Tensor}

The sums of the normal and the tangential stress-contributions are displayed in Fig. 9 for two side-pressures $p=20$ and $p=200$. The lines show the stress measured on the walls, and the symbols correspond to the stress measured via the micro-macro average in Eq. (31), proving the reasonable quality of the micro-macro transition as compared to the wall stress "measurement".

There is also other macroscopic information hidden in the stress-strain curves in Fig. 9. From the initial, rapid increase in stress, one can determine moduli of the bulk-material, i.e, the stiffness under confinement $p$. Later, the stress reaches a peak at approximately $2.6 p$ and then saturates at about $2 p$. From both peak- and saturation stress, one obtains the yield stresses at peak and in critical state flow, respectively [61].

Note that for the parameters used here, both the dynamic stress and the tangential contributions to the stress tensor are more than one order of magnitude smaller than the normal contributions. As a cautionary note, we remark also that the artificial stress induced by the background viscous force is negligible here (about two percent), when $\gamma_{b}=10^{-3} \mathrm{~kg} \mathrm{~s}^{-1}$ and a compression frequency $f=0.1 \mathrm{~s}^{-1}$ are used. For 

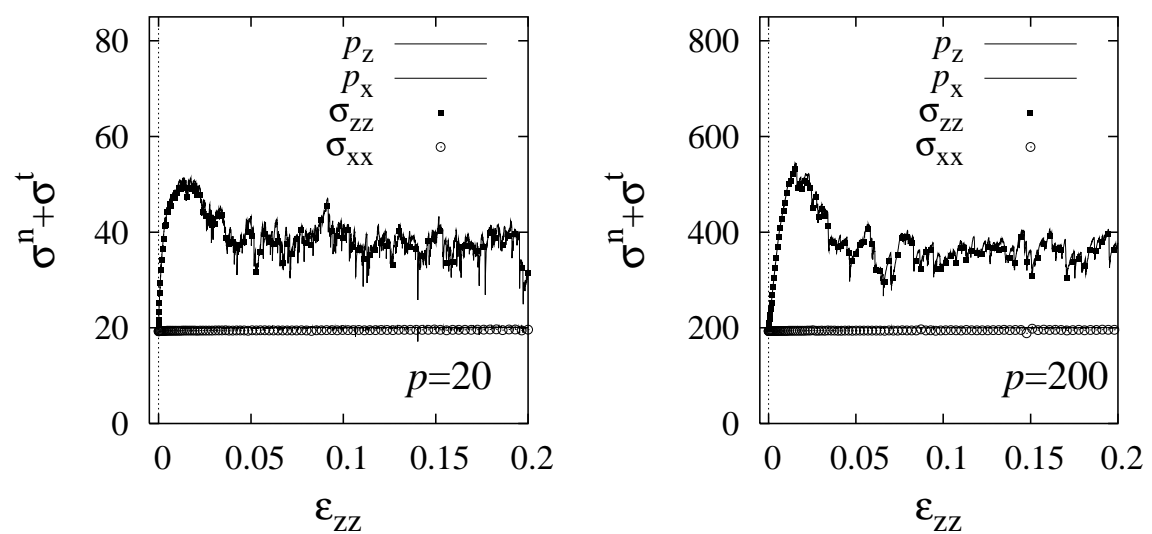

Fig. 9 Total stress tensor $\sigma=\sigma^{n}+\sigma^{t}$ for small (Left) and high (Right) pressure - the agreement between the wall pressure and the averaged stress is almost perfect.

faster compression with $f=0.5 \mathrm{~s}^{-1}$, one obtains about ten per-cent contribution to stress from the artificial background force.

The behavior of the stress is displayed in Fig. 10, where the isotropic stress $\frac{1}{2} \operatorname{tr} \sigma$ is plotted in units of $p$, and the deviatoric fraction is plotted in units of the isotropic stress. Note that the tangential forces do not contribute to the isotropic stress here since the corresponding entries in the averaging procedure compensate. From Fig. 10 , we evidence that both normal contributions, the non-dimensional trace and the non-dimensional deviator behave similarly, independent of the side pressure: Starting from an initial value, a maximum is approached, where the maximum is only weakly dependent on $p$.
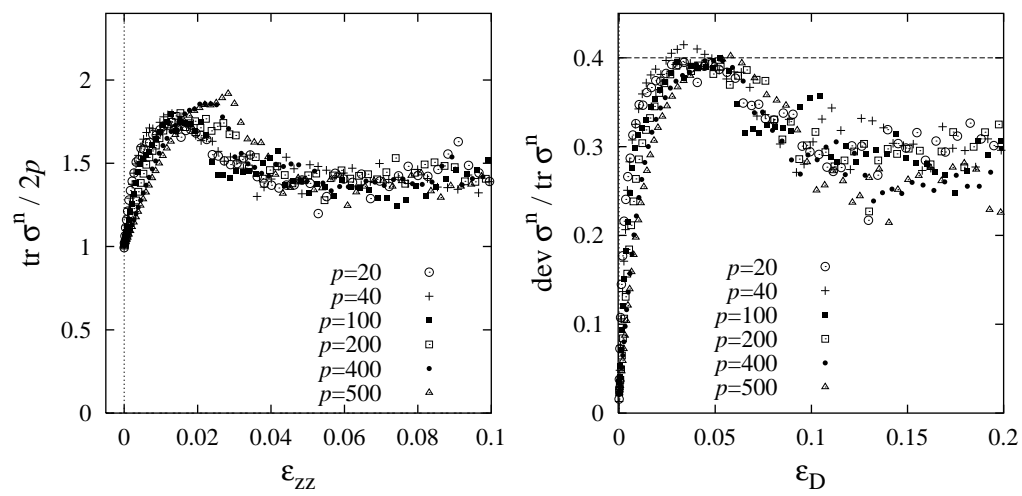

Fig. 10 Non-dimensional stress tensor contributions for different $p$. The isotropic (Left) and the deviatoric fractions (Right) are displayed as functions of the vertical and deviatoric strain, respectively. 
The increase of stress is faster for lower $p$. After the maximum is reached, the stresses decay and approach a smaller value in the critical state flow regime. Using the definitions $s_{V}:=\operatorname{tr} \sigma /(2 p)-1$ and $s_{D}:=\operatorname{dev} \sigma / \operatorname{tr} \sigma$, the maximal (nondimensional) isotropic and deviatoric stresses are $s_{V}^{\max } \approx 0.8 \pm 0.1$ and $s_{D}^{\max } \approx$ $0.4 \pm 0.02$, respectively, with a rather large error margin. The corresponding values at critical state flow are $s_{V}^{c} \approx 0.4 \pm 0.1$ and $s_{D}^{c} \approx 0.29 \pm 0.04$.

The evolution of the deviatoric stress fraction, $s_{D}$, as function of $\varepsilon_{D}$, is displayed in Fig. 10. Like the fabric, also the deviatoric stress exponentially approaches its maximum. This is described by the differential equation

$$
\frac{\partial s_{D}}{\partial \varepsilon_{D}}=\beta_{s}\left(s_{D}^{\max }-s_{D}\right),
$$

where $\beta_{s}=\beta_{s}(p)$ is decaying with increasing $p$ (roughly as $\beta_{s} \approx p^{-1 / 2}$ ). For more details on the deviatoric stress and also on the tangential contribution to the stress, see $[33,34,36,35]$.

\section{Larger Computational Examples}

In this section, several examples of rather large particle numbers simulated with DEM and ED are presented. The ED algorithm is first used to simulate a freely cooling dissipative gas in two and three dimensions $[45,56]$. Then, a peculiar three dimensional ring-shear experiment is modeled with soft sphere DEM.

\subsection{Free Cooling and Cluster Growth (ED)}

In the following, a two-dimensional system of length $L=l / d=560$ with $N=99856$ dissipative particles of diameter $d=2 a$ is examined [51, 45], with volume fraction $v=0.25$ and restitution coefficient $r=0.9$. This $2 \mathrm{D}$ system is compared to a threedimensional system of length $L=l / d=129$ with $N=512000$ dissipative spheres of diameter $d$ and volume fraction $v=0.25$ with $r=0.3$ [56].

\subsubsection{Initial configuration}

Initially the particles are arranged on a square lattice with random velocities drawn from an interval with constant probability for each coordinate. The mean total velocity, i.e. the random momentum due to the fluctuations, is eliminated in order to have a system with its center of mass at rest. The system is allowed to evolve for some time, until the arbitrary initial condition is forgotten, i.e. the density is homogeneous, and the velocity distribution is a Gaussian in each coordinate. Then 
dissipation is switched on and the evolution of the system is reported for the selected $r$. In order to avoid the inelastic collapse, the TC model is used, which reduces dissipation if the time between collisions drops below a value of $t_{c}=10^{-5} \mathrm{~s}$.
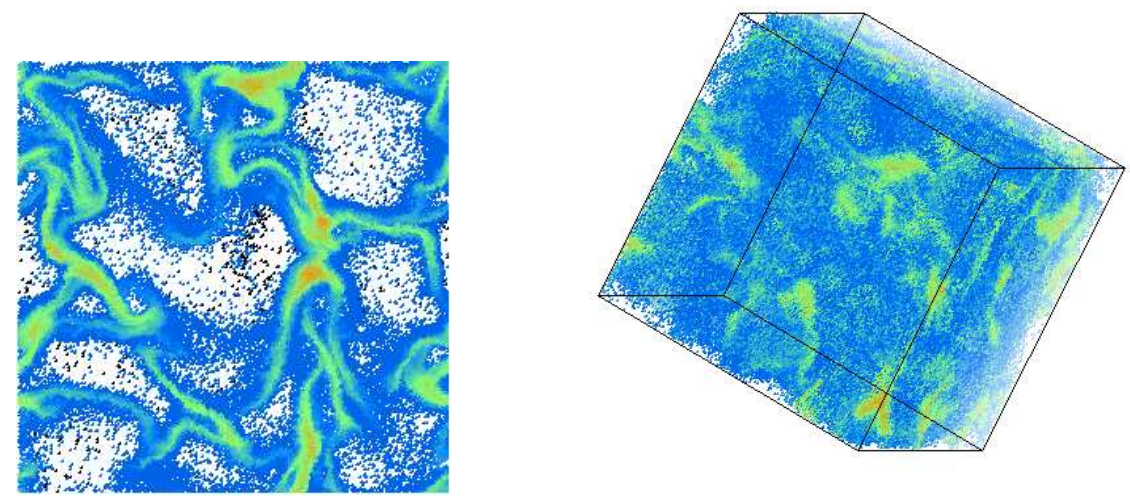

Fig. 11 (Left) Collision frequency of individual particles from a 2D simulation, after about 5200 collisions per particle. (Right) Cluster visualization from a 3D simulation. The colors in both panels indicate large (red), medium (green), and small (blue) collision rates.

\subsubsection{System evolution}

For the values of $r$ used here, the system becomes inhomogeneous quite rapidly $[45,56]$. Clusters, and thus also dilute regions, build up and have the tendency to grow. Since the system is finite, their extension will reach system size at a finite time. Thus we distinguish between three regimes of system evolution: (i) the initially (almost) homogeneous state, (ii) the cluster growth regime, and (iii) the system size dependent final stage where the clusters have reached system size. We note that a cluster does not behave like a solid body, but has internal motion and can eventually break into pieces after some time. These pieces (small clusters) collide and can merge to larger ones.

In Fig. 11, snapshots are presented and the collision rate is color-coded. The collision rate and the pressure are higher inside the clusters than at their surface. Note that most of the computational effort is spent in predicting collisions and to compute the velocities after the collisions. Therefore, the regions with the largest collision frequencies require the major part of the computational resources. Due to the TC model, this effort stays limited and the simulations can easily continue for many thousand collisions per particle. 


\subsubsection{Discussion}

Note that an event driven simulation can be 10-100 times faster than a soft-particle DEM code applied to model the same particle number. However, ED is rather limited to special, simple interactions between the particles.

\subsection{D (Ring) Shear Cell Simulation}

The simulation in this section models a ring-shear cell experiment, as recently proposed $[15,16]$. The interesting observation in the experiment is a universal shear zone, initiated at the bottom of the cell and becoming wider and moving inwards while propagating upwards in the system.

In the following, the shear-band will be examined, and the micro-macro transition from will be performed, leading to a yield stress (or flow function) based on a single simulation. This is in contrast to the two-dimensional example from the previous chapter, where the yield stress had to be determined from different simulations with different side stress $p$. In the ring shear cell, space- and time-averaging is possible, so that - at different radial and vertical positions, one obtains data for different density, stress, velocity gradient, etc.

\subsubsection{Model system}

The numerical model chosen here is DEM with smooth particles in three dimensions. In order to save computing time, only a quarter of the ring-shaped geometry is simulated. The walls are cylindrical, and are rough on the particle scale due to some attached particles. The outer cylinder wall with radius $R_{o}$, and part of the bottom $r>R_{s}$ are rotating around the symmetry axis, while the inner wall with radius $R_{i}$, and the attached bottom-disk $r<R_{s}$ remain at rest. In order to resemble the experiment, the geometry data are $R_{i}=0.0147 \mathrm{~m}, R_{s}=0.085 \mathrm{~m}$, and $R_{o}=0.110 \mathrm{~m}$. Note that the small $R_{i}$ value is artificial, but it does not affect the results for small and intermediate filling heights.

The slit in the bottom wall at $r=R_{S}$ triggers a shear band. In order to examine the behavior of the shear band as function of the filling height $H$, this system is filled with 6000 to 64000 spherical particles with mean radius $1.0 \mathrm{~mm}$ and radii range $0.5 \mathrm{~mm}<a<1.5 \mathrm{~mm}$, which interact here via repulsive and dissipative forces only. The particles are forced towards the bottom by the gravity force $f_{g}=m g$ here and are kept inside the system by the cylindrical walls. In order to provide some wall roughness, a fraction of the particles (about 3 per-cent) that are originally in contact with the walls are glued to the walls and move with them. 


\subsubsection{Material and system parameters}

The material parameters for the particle-particle and -wall interactions are $k=$ $10^{2} \mathrm{~N} / \mathrm{m}$ and $\gamma_{0}=2.10^{-3} \mathrm{~kg} / \mathrm{s}$. Assuming a collision of the largest and the smallest particle used, the reduced mass $m_{12}=2.9410^{-6} \mathrm{~kg}$, leads to a typical contact duration $t_{c}=5.410^{-4} \mathrm{~s}$ and a restitution coefficient of $r=0.83$. The integration time step is $t_{\mathrm{DEM}}=5 \cdot 10^{-6} \mathrm{~s}$, i.e. two orders of magnitude smaller than the contact duration.
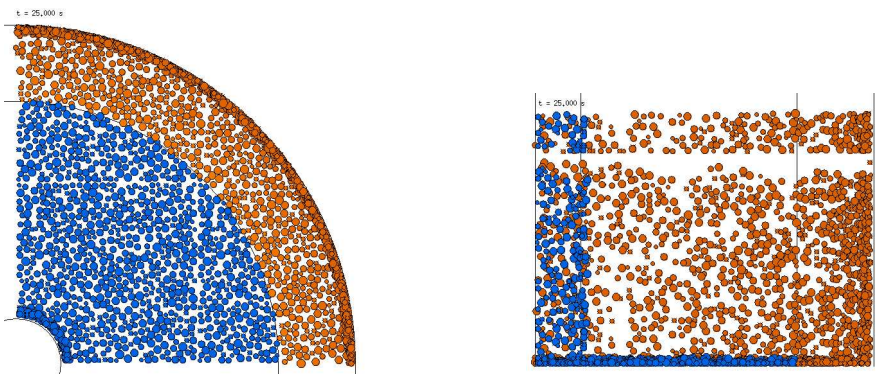

Fig. 12 Snapshots from the quarter-cylinder geometry. Visible are here only those particles glued to the wall; the cylinder and slit positions are indicated by the lines. (Left) Top-view and (Right) front-view. The colors blue and red correspond to static and moving wall particles.

The simulations run for $25 \mathrm{~s}$ with a rotation rate $f_{o}=0.01 \mathrm{~s}^{-1}$ of the outer cylinder, with angular velocity $\Omega_{o}=2 \pi f_{o}$. For the average of the displacement, only times $t>10 \mathrm{~s}$ are taken into account. Within the averaging accuracy, the system seemingly has reached a quasi-steady state after about $8 \mathrm{~s}$. The empty cell is shown in Fig. 12, while three realizations with different filling height are displayed in Fig. 13 , both as top- and front-view.

\subsubsection{Shear deformation results}

From the top-view, it is evident that the shear band moves inwards with increasing filling height, and it also becomes wider. From the front-view, the same information can be evidenced and, in addition, the shape of the shear band inside the bulk is visible: The inwards displacement happens deep in the bulk and the position of the shear band is not changing a lot closer to the surface.

In order to allow for a more quantitative analysis of the shear band, both on the top and as function of depth, we perform fits with the universal shape function proposed in [15]:

$$
\frac{v_{\varphi}(r)}{r \Omega_{o}}=A\left(1+\operatorname{erf}\left(\frac{r-R_{c}}{W}\right)\right),
$$




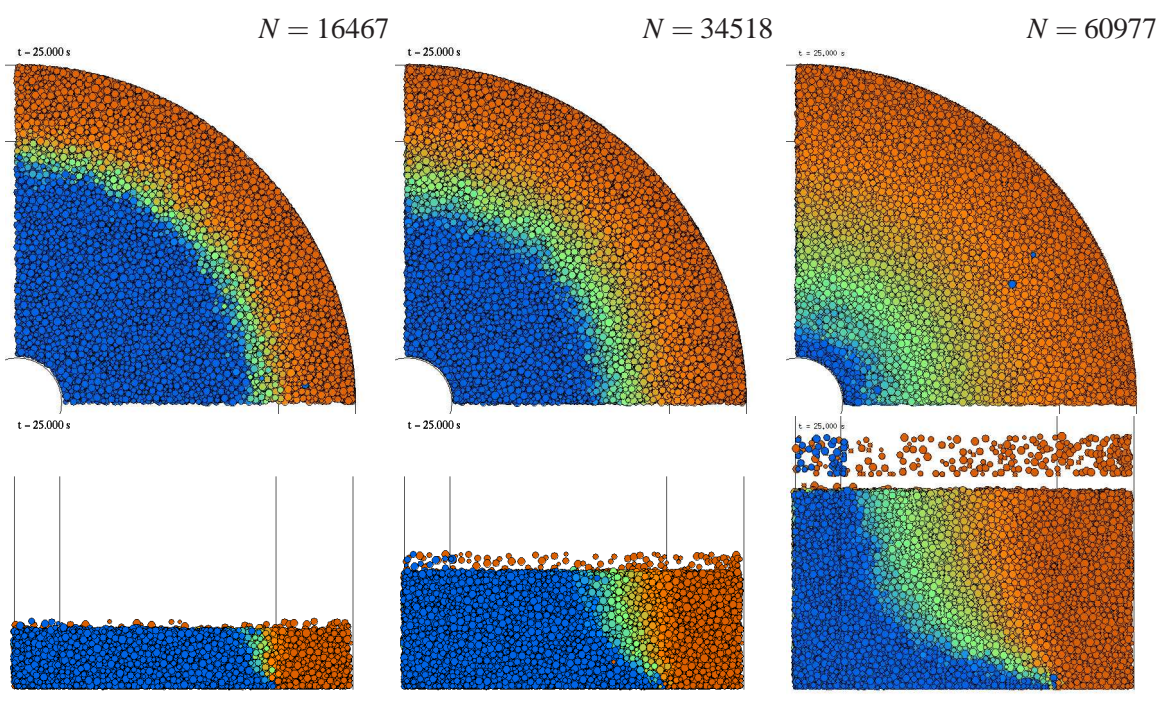

Fig. 13 Snapshots from simulations with different filling heights seen from the top and from the front, and the particle number $N$ is given in the inset. The colors blue, green, orange and red denote particles with $r \mathrm{~d} \phi \leq 0.5 \mathrm{~mm}, r \mathrm{~d} \phi \leq 2 \mathrm{~mm}, r \mathrm{~d} \phi \leq 4 \mathrm{~mm}$, and $r \mathrm{~d} \phi>4 \mathrm{~mm}$, i.e. the displacement in tangential direction per second, respectively. The filling heights in these simulations are $H=$ $0.018 \mathrm{~m}, 0.037 \mathrm{~m}$, and $0.061 \mathrm{~m}$ (from left to right).

where $A$ is a dimensionless amplitude $A=0.50 \pm 0.02, R_{C}$ is the center of the shearband, and $W$ its width.

The fits to the simulations confirm qualitatively the experimental findings in so far that the center of the shear band, as observed on top of the material, see Fig. 14 , moves inwards with a $R_{c} \propto H^{5 / 2}$ behavior, and that the width of the shear band increases almost linearly with $H$. For filling heights larger than $H \approx 0.05 \mathrm{~m}$, deviations from this behavior are observed, because the inner cylinder is reached and thus sensed by the shearband. Slower shearing does not affect the center, but reduces slightly the width - as checked by one simulation.

Like in the experiments, the behavior of the shearband within the bulk, see Fig. 15 , deviates qualitatively from the behavior seen from the top. Instead of a slow motion of the shear band center inwards, the shear band rapidly moves inwards at small heights $h$, and reaches a saturation distance with small change closer to the surface. Again, a slower rotation does not affect the center but reduces the width.

From the velocity field in the bulk it is straightforward to compute the velocity gradient tensor and, from this extracting the (symmetric) strain rate:

$$
\dot{\gamma}=\sqrt{d_{1}^{2}+d_{2}^{2}}=\frac{1}{2} \sqrt{\left(\frac{\partial v_{\phi}}{\partial r}-\frac{v_{\phi}}{r}\right)^{2}+\left(\frac{\partial v_{\phi}}{\partial z}\right)^{2}},
$$

i.e., the shear intensity in the shear plane [40]. Note that the solid-body rotation term $v_{\phi} / r$ comes from the cylindrical coordinate system used. The shear planes are 

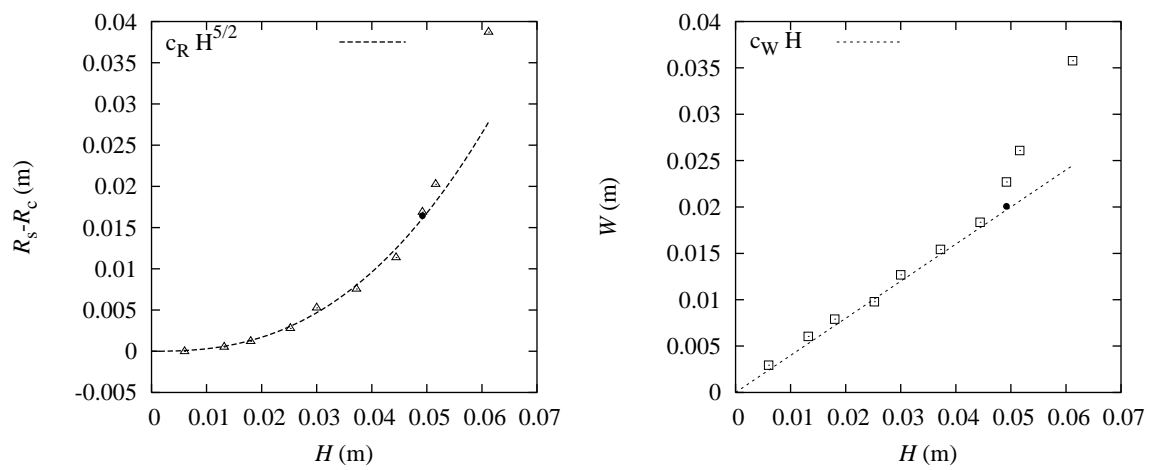

Fig. 14 (Left) Distance of the top-layer shearband center from the slit, both plotted against the filling height $H$. The open symbols are simulation results, the solid symbol is a simulation with slower rotation $f_{o}=0.005 \mathrm{~s}^{-1}$, and the line is a fit with constant $c_{R}=30$. (Right) Width of the shearband from the same simulations; the line is a fit with $c_{W}=2 / 5$.
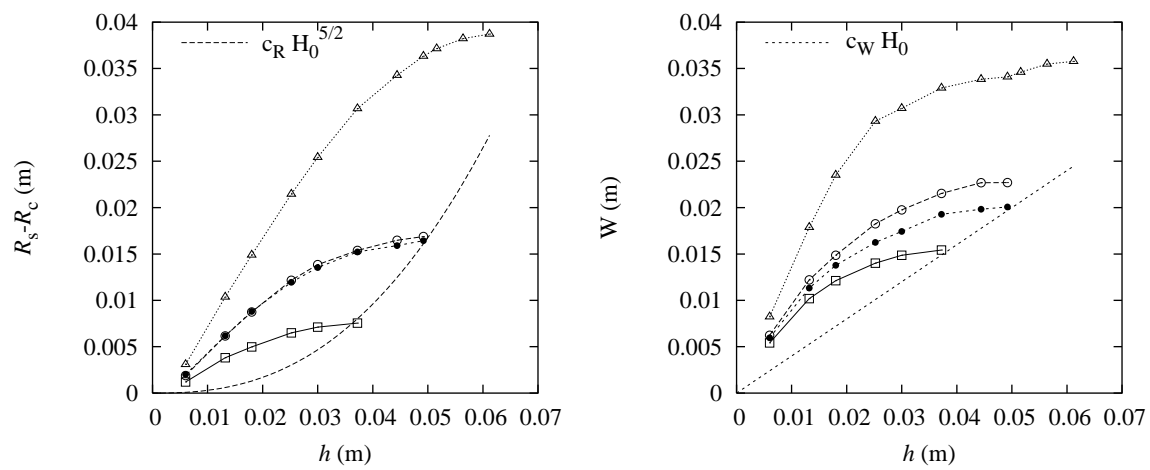

Fig. 15 (Left) Distance of the bulk shearband center from the slit and, (Right) width of the shearband, both plotted against the height $h$. The open symbols are simulation results obtained with $f_{o}=0.01 \mathrm{~s}^{-1}$, the solid symbols are obtained with slower rotation $f_{o}=0.005 \mathrm{~s}^{-1}$. Squares, circles and triangles correspond to the filling heights $H=0.037 \mathrm{~m}, 0.049 \mathrm{~m}$, and $0.061 \mathrm{~m}$, respectively. The curves are identical to those plotted in Fig. 14.

in fact described by a normal unit vector $\hat{\gamma}=(\cos \theta, 0, \sin \theta)$, with $\theta=\theta(r, z)=$ $\arccos \left(d_{1} / \dot{\gamma}\right)$, as predicted [12]. The center of the shear band indicates the direction of the unit-vector $\hat{\gamma}$. In the system with friction, we observe that the average particles spin is also normal to the shear-plane, i.e., parallel to $\hat{\gamma}$, within the rather strong fluctuations (data not shown).

From the stress, as computed according to Eq. (31), the shear stress is extracted (in analogy to the strain rate) as proposed in [12]:

$$
|\tau|=\sqrt{\sigma_{r \phi}^{2}+\sigma_{z \phi}^{2}} .
$$


Remarkably, the shear stress intensity $|\tau| / p \approx \mu$ is almost constant for practically all averaging volumina with strain rates larger than some threshold value, i.e., $\dot{\gamma}>\dot{\gamma}_{c}$, with $\dot{\gamma}_{c} \approx 0.02 \mathrm{~s}^{-1}$. Whether the threshold has a physical meaning or is only an artefact due to the statistical fluctuations in the average data has to be examined further by much longer runs with better statistics.

From the constant shear stress intensity in the shear zone, one can determine the Mohr-Coulomb-type friction angle of the equivalent macroscopic constitutive law, see Fig. 16 , as $\psi \approx \arcsin \mu$. Interestingly, without friction $\psi$ is rather large, i.e., much larger than expected from a frictionless material, whereas it is astonishingly small with friction (data not shown), i.e., smaller than the microscopic contact friction $\mu=0.4$ used, see Ref. [40].
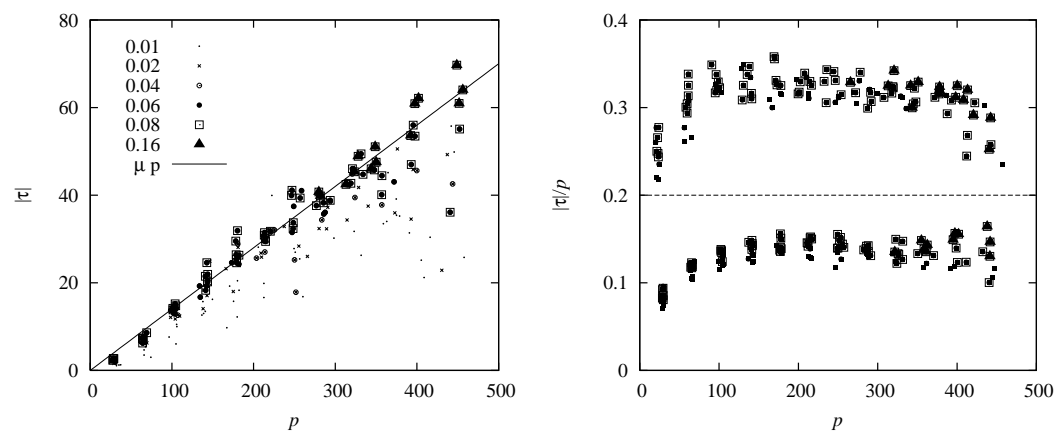

Fig. 16 (Left) Shear stress $|\tau|$ and (Right) shear stress intensity $|\tau| / p$ plotted against pressure. The size of the points is proportional to the shear rate, and the dashed line (right panel) separates the data from simulations without (Bottom) and with (Top) friction, see [40].

\subsubsection{Discussion}

In summary, the example of a ring shear cell simulation in 3D has shown, that even without the more complicated details of fancy interaction laws, experiments can be reproduced at least qualitatively. A more detailed study of quantitative agreement has been performed in 2D [27], and is in progress for the 3D case.

A challenge for the future remains the micro-macro transition, for which a first result has been shown, i.e. the yield stress can be extracted from a single 3D DEM simulation for various pressures and shear rates. Open remains an objective continuum theory formulation of the shear band problem. 


\section{Conclusion}

The present study is a summary of the most important details about soft particle molecular dynamics (MD), widely referred to as discrete element methods (DEM) in engineering, and hard particle event driven (ED) simulations, together with an attempt to link the two approaches in the dense limit where multi-particle contacts become important.

As an example for a micro-macro transition, the stress tensor was defined and computed for dynamic and quasi-static systems. This led, for example, to a global equation of state, valid for all attainable densities, and also to the partial stresses due to normal and tangential (frictional) contacts. For the latter situation, the micromacro average is compared to the macroscopic stress (=force/area) measurement (with reasonable agreement) and, at least in $3 \mathrm{D}$, a yield stress function can be extracted from a single ring shear cell simulation.

In conclusion, discrete element methods have proven a helpful tool for the understanding of many granular systems, while MD is the standard tool for atomistic and molecular systems. The methods presented in this paper can be applied to both DEM and MD simulation results with the goal to obtain micro- and particle-based constitutive relations for continuum theory.

The qualitative approach on DEM of the early years has now developed into the attempt of a quantitative predictive modeling tool for the diverse modes of complex behavior in granular media. To achieve this goal will be a research challenge for the next decades, involving enhanced kinetic theories for dense collisional flows and elaborate constitutive models for quasi-static, dense systems with shear band localisation.

In the future this tool will allow to impose a desired behavior by control or design, with particular application in mind as, e.g., modern sintered materials, reactors involving catalysts, and many others.

Acknowledgements We acknowledge the financial support of several funding institutions that supported the reviewed research, and also the helpful discussions with, and contributions from the many persons that contributed to these results.

\section{References}

1. Alam, M., Luding, S.: How good is the equipartition assumption for transport properties of a granular mixture. Granular Matter 4(3), 139-142 (2002)

2. Alam, M., Luding, S.: First normal stress difference and crystallization in a dense sheared granular fluid. Phys. Fluids 15(8), 2298-2312 (2003)

3. Alam, M., Luding, S.: Rheology of bidisperse granular mixtures via event driven simulations. J. Fluid Mech. 476, 69-103 (2003)

4. Alam, M., Willits, J.T., Arnarson, B.O., Luding, S.: Kinetic theory of a binary mixture of nearly elastic disks with size and mass-disparity. Physics of Fluids 14(11), 4085-4087 (2002)

5. Allen, M.P., Tildesley, D.J.: Computer Simulation of Liquids. Oxford University Press, Oxford (1987) 
6. van Baars, S.: Discrete element analysis of granular materials. Ph.D. thesis, Technische Universiteit Delft, Delft, Nederlands (1996)

7. Bartels, G., Unger, T., Kadau, D., Wolf, D.E., Kertesz, J.: The effect of contact torques on porosity of cohesive powders. Granular Matter 7, 139 (2005)

8. Bashir, Y.M., Goddard, J.D.: A novel simulation method for the quasi-static mechanics of granular assemblages. J. Rheol. 35(5), 849-885 (1991)

9. Cundall, P.A., Strack, O.D.L.: A discrete numerical model for granular assemblies. Géotechnique 29(1), 47-65 (1979)

10. David, C.T., Garcia-Rojo, R., Herrmann, H.J., Luding, S.: Powder flow testing with 2d and 3d biaxial and triaxial simulations. Particle and Particle Systems Characterization 24(1), 29-33 (2007)

11. David, C.T., Rojo, R.G., Herrmann, H.J., Luding, S.: Hysteresis and creep in powders and grains. In: R. Garcia-Rojo, H.J. Herrmann, S. McNamara (eds.) Powders and Grains 2005, pp. 291-294. Balkema, Leiden, Netherlands (2005)

12. Depken, M., van Saarloos, W., van Hecke, M.: Continuum approach to wide shear zones in quasistatic granular matter. Phys. Rev. E 73, 031,302 (2006)

13. Dintwa, E., van Zeebroeck, M., Tijskens, E., Ramon, H.: Torsion of viscoelastic spheres in contact. Granular Matter 7, 169 (2005)

14. Els, D.: Definition of roll velocity for spherical particles (2006). Submitted

15. Fenistein, D., van Hecke, M.: Kinematics - wide shear zones in granular bulk flow. Nature 425(6955), 256 (2003)

16. Fenistein, D., van de Meent, J.W., van Hecke, M.: Universal and wide shear zones in granular bulk flow. Phys. Rev. Lett. 92, 094,301 (2004). E-print cond-mat/0310409

17. Haff, P.K.: Grain flow as a fluid-mechanical phenomenon. J. Fluid Mech. 134, 401-430 (1983)

18. Herbst, O., Müller, P., Otto, M., Zippelius, A.: Local equation of state and velocity distributions of a driven granular gas. Phys. Rev. E 70, 051,313-1-14 (2004). E-print condmat/0402104

19. Herrmann, H.J., Hovi, J.P., Luding, S. (eds.): Physics of dry granular media - NATO ASI Series E 350. Kluwer Academic Publishers, Dordrecht (1998)

20. Hinrichsen, H., Wolf, D.E.: The Physics of Granular Media. Wiley VCH, Weinheim, Germany (2004)

21. Janssen, H.A.: Versuche über Getreidedruck in Silozellen. Zeitschr. d. Vereines deutscher Ingenieure 39(35), 1045-1049 (1895)

22. Jenkins, J.T., Richman, M.W.: Kinetic theory for plane shear flows of a dense gas of identical, rough, inelastic, circular disks. Phys. of Fluids 28, 3485-3494 (1985)

23. Kawamura, H.: A simple theory of hard disk transition. Prog. Theor. Physics 61, 1584-1596 (1979)

24. Kirkwood, J.G., Buff, F.P., Green, M.S.: The statistical mechanical theory of transport processes. J. Chem. Phys. 17(10), 988 (1949)

25. Kishino, Y. (ed.): Powders \& Grains 2001. Balkema, Rotterdam (2001)

26. Lätzel, M., Luding, S., Herrmann, H.J.: Macroscopic material properties from quasi-static, microscopic simulations of a two-dimensional shear-cell. Granular Matter 2(3), 123-135 (2000). E-print cond-mat/0003180

27. Lätzel, M., Luding, S., Herrmann, H.J., Howell, D.W., Behringer, R.P.: Comparing simulation and experiment of a 2d granular couette shear device. Eur. Phys. J. E 11(4), 325-333 (2003)

28. Lubachevsky, B.D.: How to simulate billards and similar systems. J. Comp. Phys. 94(2), 255 (1991)

29. Lubachevsky, B.D.: Simulating billiards: Serially and in parallel. Int.J. in Computer Simulation 2, 373-411 (1992)

30. Luding, S.: Surface waves and pattern formation in vibrated granular media. In: Powders \& Grains 97, pp. 373-376. Balkema, Amsterdam (1997)

31. Luding, S.: Collisions \& contacts between two particles. In: H.J. Herrmann, J.P. Hovi, S. Luding (eds.) Physics of dry granular media - NATO ASI Series E350, p. 285. Kluwer Academic Publishers, Dordrecht (1998) 
32. Luding, S.: Liquid-solid transition in bi-disperse granulates. Advances in Complex Systems 4(4), 379-388 (2002)

33. Luding, S.: Micro-macro transition for anisotropic, frictional granular packings. Int. J. Sol. Struct. 41, 5821-5836 (2004)

34. Luding, S.: Molecular dynamics simulations of granular materials. In: H. Hinrichsen, D.E. Wolf (eds.) The Physics of Granular Media, pp. 299-324. Wiley VCH, Weinheim, Germany (2004)

35. Luding, S.: Anisotropy in cohesive, frictional granular media. J. Phys.: Condens. Matter 17, S2623-S2640 (2005)

36. Luding, S.: Shear flow modeling of cohesive and frictional fine powder. Powder Technology 158, 45-50 (2005)

37. Luding, S.: About contact force-laws for cohesive frictional materials in $2 \mathrm{~d}$ and $3 \mathrm{~d}$. In: P. Walzel, S. Linz, C. Krülle, R. Grochowski (eds.) Behavior of Granular Media, pp. 137147. Shaker Verlag (2006). Band 9, Schriftenreihe Mechanische Verfahrenstechnik, ISBN 3-8322-5524-9

38. Luding, S.: Contact models for very loose granular materials. In: P. Eberhard (ed.) Symposium on Multiscale Problems in Multibody System Contacts, pp. 135-150. Springer (2007). ISBN 978-1-4020-5980-3

39. Luding, S.: Cohesive frictional powders: Contact models for tension. Granular Matter 10, 235-246 (2008)

40. Luding, S.: The effect of friction on wide shear bands. Part. Science and Technology 26(1), 33-42 (2008)

41. Luding, S., Clément, E., Blumen, A., Rajchenbach, J., Duran, J.: Anomalous energy dissipation in molecular dynamics simulations of grains: The "detachment effect". Phys. Rev. E 50, 4113 (1994)

42. Luding, S., Clément, E., Blumen, A., Rajchenbach, J., Duran, J.: The onset of convection in molecular dynamics simulations of grains. Phys. Rev. E 50, R1762 (1994)

43. Luding, S., Clément, E., Rajchenbach, J., Duran, J.: Simulations of pattern formation in vibrated granular media. Europhys. Lett. 36(4), 247-252 (1996)

44. Luding, S., Goldshtein, A.: Collisional cooling with multi-particle interactions. Granular Matter 5(3), 159-163 (2003)

45. Luding, S., Herrmann, H.J.: Cluster growth in freely cooling granular media. Chaos 9(3), 673-681 (1999)

46. Luding, S., Herrmann, H.J.: Micro-macro transition for cohesive granular media (2001). In: Bericht Nr. II-7, Inst. für Mechanik, Universität Stuttgart, S. Diebels (Ed.)

47. Luding, S., Huthmann, M., McNamara, S., Zippelius, A.: Homogeneous cooling of rough dissipative particles: Theory and simulations. Phys. Rev. E 58, 3416-3425 (1998)

48. Luding, S., Lätzel, M., Herrmann, H.J.: From discrete element simulations towards a continuum description of particulate solids. In: A. Levy, H. Kalman (eds.) Handbook of Conveying and Handling of Particulate Solids, pp. 39-44. Elsevier, Amsterdam, The Netherlands (2001)

49. Luding, S., Lätzel, M., Volk, W., Diebels, S., Herrmann, H.J.: From discrete element simulations to a continuum model. Comp. Meth. Appl. Mech. Engng. 191, 21-28 (2001)

50. Luding, S., Manetsberger, K., Muellers, J.: A discrete model for long time sintering. Journal of the Mechanics and Physics of Solids 53(2), 455-491 (2005)

51. Luding, S., McNamara, S.: How to handle the inelastic collapse of a dissipative hard-sphere gas with the TC model. Granular Matter 1(3), 113-128 (1998). E-print cond-mat/9810009

52. Luding, S., Suiker, A.: Self-healing of damaged particulate materials through sintering (2008). Submitted to Philosophical Magazine

53. Luding, S., Suiker, A., Kadashevich, I.: Discrete element modeling of self-healing processes in damaged particulate materials. In: A.J.M. Schmets, S. van der Zwaag (eds.) Proceedings of the 1st International Conference on Self Healing Materials. Springer series in Material Science, Berlin, Germany (2007). ISBN 978-1-4020-6249-0

54. McNamara, S., Young, W.R.: Inelastic collapse in two dimensions. Phys. Rev. E 50(1), R28R31 (1994) 
55. Miller, S.: Clusterbildung in granularen gasen. Ph.D. thesis, Universität Stuttgart (2004)

56. Miller, S., Luding, S.: Cluster growth in two- and three-dimensional granular gases. Phys. Rev. E 69, 031,305 (2004)

57. Miller, S., Luding, S.: Event driven simulations in parallel. J. Comp. Phys. 193(1), 306-316 (2004)

58. Pöschel, T., Luding, S. (eds.): Granular Gases. Springer, Berlin (2001). Lecture Notes in Physics 564

59. Rapaport, D.C.: The Art of Molecular Dynamics Simulation. Cambridge University Press, Cambridge (1995)

60. Sadd, M.H., Tai, Q.M., Shukla, A.: Contact law effects on wave propagation in particulate materials using distinct element modeling. Int. J. Non-Linear Mechanics 28(2), 251 (1993)

61. Schwedes, J.: Review on testers for measuring flow properties of bulk solids. Granular Matter 5(1), 1-45 (2003)

62. Sperl, M.: Experiments on corn pressure in silo cells. translation and comment of janssen's paper from 1895. Granular Matter 8(2), 59-65 (2006)

63. Thornton, C.: Numerical simulations of deviatoric shear deformation of granular media. Géotechnique 50(1), 43-53 (2000)

64. Thornton, C., Antony, S.J.: Quasi-static deformation of a soft particle system. Powder Technology 109(1-3), 179-191 (2000)

65. Thornton, C., Zhang, L.: A dem comparison of different shear testing devices. In: Y. Kishino (ed.) Powders \& Grains 2001, pp. 183-190. Balkema, Rotterdam (2001)

66. Tighe, B.P., Sperl, M.: Pressure and motion of dry sand: translation of hagen's paper from 1852. Granular Matter 9(3/4), 141-144 (2007)

67. Tomas, J.: Particle adhesion fundamentals and bulk powder consolidation. KONA 18, 157169 (2000)

68. Vermeer, P.A., Diebels, S., Ehlers, W., Herrmann, H.J., Luding, S., Ramm, E. (eds.): Continuous and Discontinuous Modelling of Cohesive Frictional Materials. Springer, Berlin (2001). Lecture Notes in Physics 568

69. Walton, O.R., Braun, R.L.: Viscosity, granular-temperature, and stress calculations for shearing assemblies of inelastic, frictional disks. J. Rheol. 30(5), 949-980 (1986)

70. Zhu, C.Y., Shukla, A., Sadd, M.H.: Prediction of dynamic contact loads in granular assemblies. J. of Applied Mechanics 58, 341 (1991) 